\title{
On the Fourier extension of non-periodic functions
}

\author{
Daan Huybrechs
}

Report TW 534, January 2009

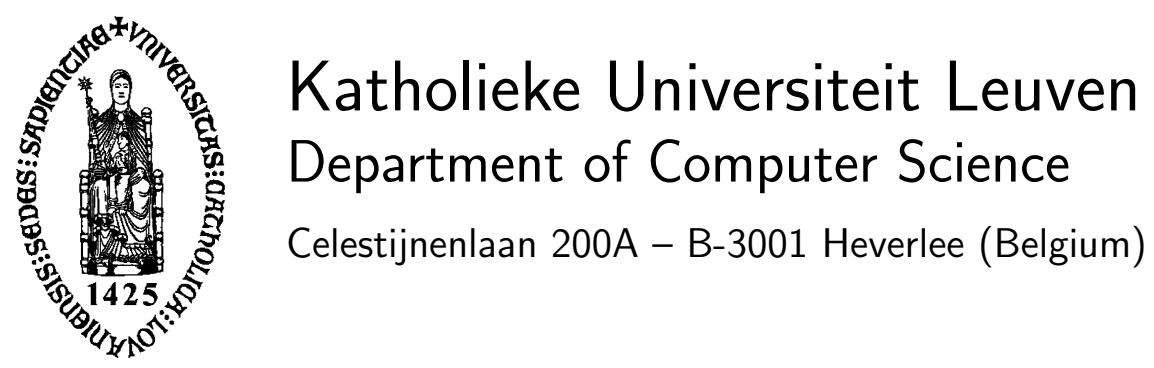




\title{
On the Fourier extension of non-periodic functions
}

\author{
Daan Huybrechs \\ Report TW 534, January 2009 \\ Department of Computer Science, K.U.Leuven
}

\begin{abstract}
We obtain exponentially accurate Fourier series for non-periodic functions on the interval $[-1,1]$ by extending these functions to periodic functions on a larger domain. The series may be evaluated, but not constructed, by means of the FFT. A complete convergence theory is given based on orthogonal polynomials that resemble Chebyshev polynomials of the first and second kinds. We analyze a previously proposed numerical method, which is unstable in theory but stable in practice. We propose a new numerical method that is stable both in theory and in practice.
\end{abstract}

Keywords : Fourier series, orthogonal polynomials, frames, numerical integration

AMS(MOS) Classification : Primary : 42A10, Secondary : 42C15, 65D32 


\title{
On the Fourier extension of non-periodic functions
}

\author{
Daan Huybrechs ${ }^{*}$
}

\begin{abstract}
We obtain exponentially accurate Fourier series for non-periodic functions on the interval $[-1,1]$ by extending these functions to periodic functions on a larger domain. The series may be evaluated, but not constructed, by means of the FFT. A complete convergence theory is given based on orthogonal polynomials that resemble Chebyshev polynomials of the first and second kinds. We analyze a previously proposed numerical method, which is unstable in theory but stable in practice. We propose a new numerical method that is stable both in theory and in practice.
\end{abstract}

\section{Introduction}

Computing a Fourier series by means of the FFT is the method of choice for approximating smooth and periodic functions. The method is stable and well understood and it yields spectral convergence. The picture changes completely when the function involved is either not smooth or not periodic, due to the presence of the well known Gibbs phenomenon. Equivalent symptoms are the lack of pointwise convergence, the slow decay of the Fourier coefficients and spurious oscillations near the points of disconinuity or near the boundaries. Due to the importance of Fourier series and the FFT, many ways have been devised to circumvent or to ameliorate the Gibbs phenomenon. We briefly recall three popular possibilities. A first approach is to filter out the oscillations (see, e.g., [22] and references therein). A second approach is to introduce a periodizing transformation and to compute the Fourier series of the resulting function. A popular choice of transformation leads to Chebyshev polynomials, which will be briefly recalled later in this paper. A third approach is to reconstruct a nonperiodic function from its truncated Fourier series by re-expanding that series into a basis of Gegenbauer polynomials [12]. Though the Fourier series itself is an inaccurate approximation, spectral accuracy can be achieved in the recovery.

All these approaches are successful and well understood. The topic of this paper is an intriguing alternative approach that has also proved to be successful, but which has not yet received as much attention in literature.

Consider a function $f \in L_{[-1,1]}^{2}$ that is not necessarily smooth or periodic. In this paper we will focus mostly on the lack of periodicity of $f$, assuming sufficient smoothness except where noted otherwise. The canonical example is

\footnotetext{
*Department of Computer Science, Katholieke Universiteit Leuven, Belgium (daan.huybrechs@cs.kuleuven.be)

${ }^{\dagger}$ The author is a Postdoctoral Fellow of the Research Foundation - Flanders (FWO).
} 


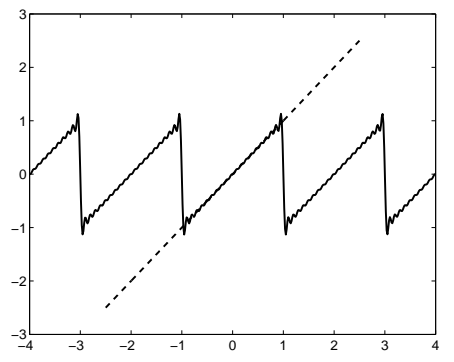

(a) Fourier series of $f$

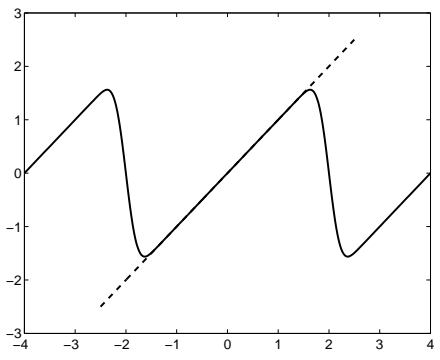

(b) A smooth and periodic extension of $f$

Figure 1: Illustration of the Fourier series of $f(x)=x$ on $[-1,1]$ with 10 terms (left panel) and a smooth extension of $f$ with period 4 rather than period 2 (right panel).

$f(x)=x$. A simple idea to obtain a spectrally accurate Fourier series is to extend the function $f$ to a function $g$ that is periodic on a larger interval, say $[-T, T]$ with $T>1$. If a suitable smooth and periodic function $g$ exists then the Fourier series of $g$ converges pointwise to the values of $g$ on $[-T, T]$. This implies by construction that the Fourier series also converges pointwise to the values of $f$ on the interval $[-1,1]$. The resulting approximation to $f$ has the interesting property that it is a classical Fourier series: we may use the FFT to evaluate it. Figure 1 illustrates the Gibbs phenomenon for $f(x)=x$ on $[-1,1]$ and a smooth extension of $f(x)$ with period 4 rather than 2 .

Note the important distinction between a smooth extension of $f$, such as the periodic function $g$ we are looking for, and the analytic continuation of $f$ in the sense of complex analysis. The latter is unique, if it exists, and has many interesting properties, but it is in general not periodic on a larger domain and therefore not of immediate interest to us. This means that, even for analytic $f$, the function $g$ is in general only infinitely differentiable and not analytic. This has implications for the possible convergence rate of the Fourier series of $g$. The Fourier series of an infinitely differentiable periodic function with $n$ terms converges superalgebraically, i.e., faster than any inverse power of $n$. The Fourier series of an analytic and periodic function on the other hand converges exponentially, i.e., $O\left(e^{-c n}\right)$ with $c>0$. One would therefore reasonably expect only superalgebraic convergence in our setting.

The question of the existence of a suitable function $g$ is quickly settled. Whitney showed that a continuous function on a bounded domain $A \subset \mathbb{R}^{d}$ can be extended to a function that is analytic on $\mathbb{R}^{d} \backslash A[26]$. This problem was thereafter called Whitney's extension problem. Hestenes gave a constructive method to extend differentiable functions [13]. Recently, Fefferman proved in great generality the existence of extensions that can be bounded in terms of $f$ in suitable norms [9]. This is an important issue for numerical stability - loosely speaking, we wish to avoid cases where $g$ is 'large' compared to $f$ - to which we will return in greater detail later on. Many constructive extension methods have been devised since the Hestenes extension. Any smooth extension of $f$ can lead to a smooth and periodic extension using cut-off functions $[3,4]$. This leads 
directly to rapidly converging Fourier series. However, this approach does not lead to optimal results and we will not further pursue the explicit construction of a smooth extension of $f$ in this manner.

A simple and useful criterion to find a suitable function $g$ was explored by Boyd in [3] and by Bruno, Hana and Pohlman in [5]. These authors proposed to solve the following optimization problem. ${ }^{1}$

Problem 1.1. For $T>1$, let $G_{n}$ be the space of $2 T$-periodic functions of the form

$$
g \in G_{n}: g(x)=\frac{a_{0}}{2}+\sum_{k=1}^{n} a_{k} \cos \frac{\pi}{T} k x+b_{k} \sin \frac{\pi}{T} k x .
$$

The Fourier extension of $f$ to the interval $[-T, T]$ is the solution to the optimization problem

$$
g_{n}:=\arg \min _{g \in G_{n}}\|f-g\|_{L_{2}[-1,1]} .
$$

The function $g_{n}$ is found as the closest fit to $f$ on $[-1,1]$ in a least squares sense. The authors mentioned above observed experimentally that the solution to this problem converges rapidly to $f$ pointwise in $[-1,1]$, including in the endpoints. However, they also observed that the Fourier coefficients $a_{k}$ and $b_{k}$ of $g_{n}$ may become very large, resulting in possible loss of precision in computations. Though the numerical algorithms involved are simple and the results obtained in these references are very encouraging, the apparent unboundedness of the Fourier coefficients of $g_{n}$ appears to be problematic.

In this paper, we analyze Problem 1.1 and two numerical methods for solving it. We restrict ourselves to the case $T=2$, which appears to be the most promising case. In this setting we prove existence and uniqueness of the solution. Moreover, we characterize the solution constructively in terms of two families of orthogonal polynomials that are related to Chebyshev polynomials of the first and second kinds. We prove that the solution converges to $f$ at a rate that is bounded independently of $f$ in most cases but that is exponential rather than superalgebraic.

These results contradict our earlier intuition, concerning the convergence rate of our Fourier series. How can the Fourier series $g_{n}$ converge exponentially if $g$ is not analytic? The answer to this apparent paradox is the observation that the functions $g_{n}$ do not converge to a fixed function $g$. In fact, we will see that the functions $g_{n}$ are unbounded for increasing $n$ outside the interval $[-1,1]$.

Unfortunately, this unbounded growth of $g_{n}$ is problematic for practical use, since it gives rise to large Fourier coefficients. It is not numerically stable to represent the exact solution in the form (1). We explore two ways to remedy this: by representing the exact solution in a different form and by representing an approximate solution in the form (1).

The exact solution to Problem 1.1 can be computed in a stable manner in a basis that is related to orthogonal polynomials through a Chebyshev-like transformation. In a typical Chebyshev approximation, one approximates $f(x)$ in a basis of Chebyshev polynomials, which is equivalent to approximating the

\footnotetext{
${ }^{1}$ The problem is called Fourier continuation by Bruno, Hana and Pohlman in [5] and Fourier extension of the third kind by Boyd in [3]. Fourier extension of the first kind is a problem where $f$ is known explicitly outside the interval $[-1,1]$. Fourier extension of the second kind is a problem where $f$ is also known outside $[-1,1]$, but it has singularities there.
} 
transformed function $f(\cos \theta)$ by its Fourier series. Here, the situation is reversed. We approximate $f$ by a Fourier series, and show that this is equivalent to approximating a transformed function in a basis of polynomials. This means that in our setting the Fourier series converges to $f$ itself, not to some transformation of $f$. This has advantages in resolving oscillations when approximating oscillatory functions: the oscillations are not warped by a transformation.

The second remedy is computing an approximate solution to Problem 1.1 in the form (1). Numerical least squares methods were previously used for this problem in $[3,5]$ and are surprisingly effective. We show that a simple numerical method for solving Problem 1.1 with $T=2$ actually yields bounded Fourier coefficients. In fact, the $l_{2}$-norm of the coefficients converges to the optimal value $\frac{1}{\sqrt{2}}\|f\|_{L_{[-1,1]}^{2}}$. This is, of course, not the exact solution anymore. Yet, the computed solution, a classical finite Fourier series, is a highly accurate approximation to $f$ on the interval $[-1,1]$. Though Problem 1.1 is ill-conditioned, we arrive in this paper at the surprising conclusion that numerical error stabilizes the computations without sacrificing accuracy. The advantage of this approach, compared to computing the exact solution, is that the form (1) is maintained and, therefore, that FFT may be used to evaluate the approximation.

In any case, exact or approximate solution, the behavior of the functions $g_{n}$ outside $[-1,1]$ does not appear to be of practical use unless $n$ is small. The exact solution is unbounded outside $[-1,1]$, the numerical solution is typically wildly oscillatory. Alternative optimization criteria can be devised that enforce smoothness of $g_{n}$ or, equivalently, decay rates of its Fourier coefficients. It appears that only algebraic convergence rates can be achieved in this setting. We do not pursue this option further in this paper.

The structure of the paper is as follows. In $\S 2$ we briefly recall Chebyshev polynomials and the concept of frames in approximation theory. We analyze Problem 1.1 in $\S 3$. We find the exact solution and derive some of its properties. We analyze numerical least squares methods for Problem 1.1 in $\S 4$. Finally, we describe a stable numerical method to compute the exact solution in $\S 5$. We end with some concluding remarks in $\S 6$.

\section{Preliminaries}

We briefly recall some properties of Chebyshev polynomials and the concept of frames in approximation theory.

\subsection{Chebyshev polynomials}

Chebyshev polynomials $T_{k}(y)$ of the first kind are classical polynomials. They are orthogonal with respect to the weight function $\frac{1}{\sqrt{1-y^{2}}}$ on the interval $[-1,1]$, and normalized such that $T_{k}(1)=1$. They arise as solutions to the Chebyshev differential equation. Alternatively, they are completely characterized by the following property:

$$
\cos k \theta=T_{k}(\cos \theta), \quad k \in \mathbb{N},
$$

which expresses the fact that $\cos k \theta$ is a polynomial in $\cos \theta$.

Chebyshev polynomials $U_{k}(x)$ are orthogonal with respect to the weight function $\sqrt{1-y^{2}}$ on the interval $[-1,1]$, and normalized such that $U_{k}(1)=k+1$. 
The counterpart of (3) is the property

$$
\frac{\sin (k+1) \theta}{\sin \theta}=U_{k}(\cos \theta), \quad k \in \mathbb{N},
$$

which expresses the fact that $\sin (k+1) \theta$ is also a polynomial in $\cos \theta$, up to a factor $\sin \theta$.

Chebyshev polynomials of the first kind are ideally suited to study polynomial approximations. For an in-depth discussion, we refer the reader to [23] and references therein, in particular [20]. In the following, we borrow the notation and results of [23].

The expansion of a function $f \in L_{[-1,1]}^{2}$ in Chebyshev polynomials of the first kind can be written as

$$
f_{n}(y)=\sum_{k=0}^{a_{n}}{ }^{\prime} a_{k} T_{k}(y)
$$

(with' indicating that the first term of the sum should be halved) where

$$
a_{k}=\frac{2}{\pi} \int_{-1}^{1} f(y) T_{k}(y) \frac{1}{\sqrt{1-y^{2}}} \mathrm{~d} y .
$$

Several bounds are given in [23] for the approximation error $E_{n}^{T}=\left\|f-f_{n}\right\|$. We focus on the case where $f$ is analytic.

Theorem 2.1. [23, Th 4.3] If $f$ is analytic with $|f(z)| \leq M$ in the region bounded by the ellipse with foci \pm 1 and major and minor semiaxis lengths summing to $\rho>1$, then for each $n \geq 0$,

$$
E_{n}^{T} \leq \frac{2 M}{(\rho-1) \rho^{n}} .
$$

The same bound holds pointwise in $[-1,1]$ (see [2]). This error differs from the error of the best polynomial approximation to $f$ in the infinity norm $\|\cdot\|_{\infty}$ only by a logarithmic factor [23].

\subsection{Bases and frames}

Let $\left\{e_{n}\right\}$ be an orthonormal basis of a separable Hilbert space $H$ with inner product $<\cdot, \cdot\rangle$. Then Parseval's identity holds:

$$
\forall f \in H:\|f\|^{2}=\sum_{k=0}^{\infty}\left|<f, e_{k}>\right|^{2} .
$$

A frame for $H$ is a set $\left\{u_{n}\right\}$ such that

$$
\forall f \in H: A\|f\|^{2} \leq \sum_{k=0}^{\infty}\left|<f, u_{k}>\right|^{2} \leq B\|f\|^{2}
$$

The constants $0<A \leq B<\infty$ are called the frame bounds. A frame is called tight if $A=B$. Frames generalize bases, since condition (7) is considerably weaker than (6). A frame may therefore not be a basis, though frames are 
always complete. For example, the functions $u_{n}$ may be linearly dependent. In general, there may be infinitely many ways to represent $f \in H$ as a linear combination of $u_{k}$.

One representation of $f$ has additional interesting properties. To any frame $\left\{u_{k}\right\}$ corresponds a dual frame $\left\{u_{k}^{*}\right\}$, with frame bounds $1 / B$ and $1 / A$, such that the series

$$
L^{*} f:=\sum_{k=0}^{\infty}<f, u_{k}^{*}>u_{k}
$$

converges to $f$ (in the norm of $H$ ). This representation is optimal in the sense that the coefficients minimize the energy among all coefficient sequences $\left\{a_{k}\right\}$ such that $f=\sum_{k} a_{k} u_{k}$, i.e.,

$$
\sum_{k=0}^{\infty}\left|<f, u_{k}^{*}>\right|^{2} \leq \sum_{k=0}^{\infty}\left|a_{k}\right|^{2}
$$

with equality if and only if $a_{k}=<f, u_{k}^{*}>$.

For a tight frame, the dual frame is proportional to the frame itself: $u_{k}^{*}=$ $\frac{1}{A} u_{k}$. It follows in this case from the frame property (7) with $A=B$ that

$$
\sum_{k=0}^{\infty}\left|<f, u_{k}^{*}>\right|^{2}=\frac{1}{A^{2}} \sum_{k=0}^{\infty}\left|<f, u_{k}>\right|^{2}=\frac{\|f\|^{2}}{A} .
$$

If all elements of a tight frame are normalized, then $A$ can be interpreted as a measure of the redundancy in the frame.

Frames were originally studied in the context of non-harmonic Fourier series [8], which resembles the setting of this paper. They were picked up and studied intensively later in the field of wavelets. For a discussion with proofs of the properties above, we refer the reader to [6].

\section{Analysis of the least squares problem}

\subsection{The function space $G_{n}$}

The Fourier extension problem was already formulated in the introduction in Problem 1.1. We are looking for a Fourier series $g_{n}$ on $[-T, T]$ of the form (1), but with the intention of performing all computations in the interval $[-1,1]$. Let us start by examining the set of Fourier functions $\mathrm{e}^{\mathrm{i} \frac{\pi}{T} x}$ in $L_{[-1,1]}^{2}$. We alter the constant function in this set in order to state the following result.

Lemma 3.1. For $T>1$, the set

$$
\Phi:=\left\{\frac{1}{\sqrt{2}}\right\} \cup\left\{e^{i \frac{\pi}{T} k x}\right\}_{k \in \mathbb{Z} \backslash 0}
$$

is a tight frame for $L_{[-1,1]}^{2}$ with frame bound $T$.

Proof. For any function $f \in L_{[-1,1]}^{2}$, we should show that $\sum_{k \in \mathbb{Z}} a_{k}^{2}=T\|f\|^{2}$, where

$$
a_{k}=\int_{-1}^{1} f(x) \mathrm{e}^{\mathrm{i} \frac{\pi}{T} k x} \mathrm{~d} x=T \int_{-\frac{1}{T}}^{\frac{1}{T}} f(T x) \mathrm{e}^{\mathrm{i} k x} \mathrm{~d} x, \quad k \in \mathbb{Z} \backslash 0,
$$


and

$$
a_{0}=\int_{-1}^{1} f(x) \frac{1}{\sqrt{2}} \mathrm{~d} x=T \int_{-\frac{1}{T}}^{\frac{1}{T}} f(T x) \frac{1}{\sqrt{2}} \mathrm{~d} x .
$$

Define the function $h(x)=T f(T x)$ for $x \in\left[-\frac{1}{T}, \frac{1}{T}\right]$ and $h(x)=0$ otherwise. We find that

$$
a_{k}=\int_{-\frac{1}{T}}^{\frac{1}{T}} h(x) \mathrm{e}^{i k x} \mathrm{~d} x=\int_{-1}^{1} h(x) \mathrm{e}^{i k x} \mathrm{~d} x, \quad k \in \mathbb{Z} \backslash 0,
$$

and

$$
a_{0}=\int_{-1}^{1} h(x) \frac{1}{\sqrt{2}} \mathrm{~d} x .
$$

It follows by Parseval's identity that $\|h\|^{2}=\sum_{k} a_{k}^{2}$. We also find that

$$
\|h\|^{2}=\int_{-1}^{1} h(x)^{2} \mathrm{~d} x=\int_{-\frac{1}{T}}^{\frac{1}{T}} T^{2} f(T x)^{2} \mathrm{~d} x=T \int_{-1}^{1} f(x)^{2} \mathrm{~d} x=T\|f\|^{2},
$$

from which the result follows.

The lemma shows that the set of Fourier functions on $[-T, T]$, restricted to the interval $[-1,1]$, is redundant. We can expect severe conditioning problems when performing computations with this frame, such as interpolation, since some functions in the set may be (close to) linearly dependent.

The case $T=2$ leads to more structure and also to more insight. For future use, we define two sets $C_{n}$ and $S_{n}$ by

$$
C_{n}:=\left\{\frac{1}{\sqrt{2}}\right\} \cup\left\{\cos k \frac{\pi}{T} x\right\}_{k=1}^{n} \quad \text { and } \quad S_{n}:=\left\{\sin k \frac{\pi}{T} x\right\}_{k=1}^{n} .
$$

Note that $C_{n}$ consists of even functions and $S_{n}$ consists of odd functions. The function space $G_{n}$ is the span of

$$
D_{n}:=C_{n} \cup S_{n} .
$$

We already established that $D_{\infty}$ is a tight frame with frame bound 2. But the functions in this set have additional interesting properties.

Lemma 3.2. For $T=2$, the set $D_{\infty}$ consists of all eigenfunctions of the Laplacian on $[-1,1]$ subject to either homogeneous Dirichlet or Neumann boundary conditions.

Proof. Distinguishing between even and odd values of the index $k$ for $T=2$ in (12) shows that the set $D_{\infty}$ can be split into the sets

$$
L^{N}:=\left\{\frac{1}{\sqrt{2}}\right\} \cup\{\cos \pi k x\}_{k=1}^{\infty} \cup\left\{\sin \pi\left(k+\frac{1}{2}\right) x\right\}_{k=0}^{\infty},
$$

and

$$
L^{D}:=\left\{\cos \pi\left(k+\frac{1}{2}\right) x\right\}_{k=0}^{\infty} \cup\{\sin \pi k x\}_{k=1}^{\infty} .
$$

The former set consists of all eigenfunctions of the Laplacian on $[-1,1]$ subject to homogeneous Neumann boundary conditions, the latter of all eigenfunctions of the Laplacian subject to homogeneous Dirichlet boundary conditions [16]. 
Both sets $L^{N}$ and $L^{D}$ are orthonormal bases for $L_{[-1,1]}^{2}$. From this observation it also follows that $D_{\infty}$ is a tight frame with frame bound $A=2$. The approximation of functions in the basis $L^{N}$ of eigenfunctions obeying Neumann boundary conditions was the subject of a systematic study in [16], with generalization to higher order differential operators in [17], generalization to higher dimensions in [18] and techniques for convergence acceleration in [14]. ${ }^{2}$ The link with eigenfunctions of the Laplacian is very fruitful and, in the remainder of this paper, we therefore restrict ourselves to the case $T=2$.

The subject of finding representations in overcomplete sets has seen a lot of activity in recent years. The concatenation of two orthonormal bases was studied in Matching Pursuit and is relevant as well in compressed sensing (see [7] and references therein). However, in that setting, it is desirable to have two bases that are as different, or as little coherent, as possible. In our setting however, both orthonormal bases are highly similar, which leads to very different analysis.

\subsection{An orthonormal basis on $[-1,1]$}

We found that $D_{\infty}$ is not a basis for $L_{[-1,1]}^{2}$ but a tight frame, which has redundancy. However, for any finite $n$, the set $D_{n}$ is a basis for a finite dimensional subspace of $L_{[-1,1]}^{2}$, i.e., all functions in the set are linearly independent. It makes perfect sense, therefore, to look for an orthonormal basis on $[-1,1]$. The orthogonalization problem naturally divides into two problems, since the even functions in $C_{n}$ and the odd functions in $S_{n}$ are automatically orthogonal to each other on the symmetric interval $[-1,1]$.

\subsubsection{Even functions}

We consider the even functions first. Denote by

$$
\mathcal{C}_{n}:=\operatorname{span} C_{n}
$$

the $(n+1)$-dimensional space spanned by the cosine functions. We start with the basic, but crucial observation that, since $\cos k x=T_{k}(\cos x)$ is a polynomial in $\cos x$ of degree $k$, it is also true that

$$
\cos k \frac{\pi}{2} x=T_{k}\left(\cos \frac{\pi}{2} x\right)
$$

is a polynomial in $\cos \frac{\pi}{2} x$ of degree $k$. This means that the orthogonalization can be expressed in terms of orthogonal polynomials.

Theorem 3.3. Let $T_{k}^{h}(y)$ be the unique normalized sequence of orthogonal polynomials satisfying

$$
\frac{4}{\pi} \int_{0}^{1} T_{k}^{h}(y) y^{l} \frac{1}{\sqrt{1-y^{2}}} \mathrm{~d} y=\delta_{k-l}, \quad l=0, \ldots, k-1 .
$$

Then the set $\left\{T_{k}^{h}\left(\cos \frac{\pi}{2} x\right)\right\}_{k=0}^{n}$ is an orthonormal basis for $\mathcal{C}_{n}$ on $[-1,1]$.

\footnotetext{
${ }^{2}$ We like to remark here that the current paper grew out of the idea of combining these two orthonormal bases, based on experience with the properties of expansions in $L^{N}$ and $L^{D}$ separately. We will not expand on that point of view in this paper, but present our results in the context of Fourier extensions.
} 
Proof. Consider a function $g \in \mathcal{C}_{n}$. Since $g$ is necessarily even, we can restrict ourselves to the interval $[0,1]$. The transformation $y=\cos \frac{\pi}{2} x$ maps the interval $[0,1]$ to itself and is invertible with $x=\frac{2}{\pi} \cos ^{-1} y$. It follows from the Chebyshev polynomial property (14) that $g\left(\frac{2}{\pi} \cos ^{-1} y\right)$ is a polynomial in $y$ on $[0,1]$, which we denote by $P_{g}(y)$.

Conversely, from (14) it also follows that each polynomial $p(y)$ of degree less than or equal to $n$ corresponds to a function $p\left(\cos \frac{\pi}{2} x\right) \in \mathcal{C}_{n}$, because the set of Chebyshev polynomials of the first kind up to degree $n$ is a basis for the space of all polynomials up to degree $n$.

We enforce orthogonality of $g$ to the function $\tilde{g} \in \mathcal{C}_{n-1}$. This means that

$$
\begin{aligned}
\int_{-1}^{1} g(x) \tilde{g}(x) \mathrm{d} x & =2 \int_{0}^{1} g(x) \tilde{g}(x) \mathrm{d} x \\
& =2 \int_{1}^{0} g\left(\frac{2}{\pi} \cos ^{-1} y\right) \tilde{g}\left(\frac{2}{\pi} \cos ^{-1} y\right) \frac{-2}{\pi} \frac{1}{\sqrt{1-y^{2}}} \mathrm{~d} y \\
& =\frac{4}{\pi} \int_{0}^{1} P_{g}(y) P_{\tilde{g}}(y) \frac{1}{\sqrt{1-y^{2}}} \mathrm{~d} y \\
& =0 .
\end{aligned}
$$

If $g(x)=T_{n}^{h}\left(\cos \frac{\pi}{2} x\right)$, then $P_{g}(y)=T_{n}^{h}(y)$ and $g(x)$ is orthogonal to all functions in $\mathcal{C}_{n-1}$. The normalization implicit in (15) corresponds exactly to the normalization of $T_{k}^{h}\left(\cos \frac{\pi}{2} x\right)$ in $L_{[-1,1]}^{2}$. This proves the result.

Note that the polynomials $T_{n}^{h}(x)$ have the same weight function as Chebyshev polynomials of the first kind, but they are orthogonal on the interval $[0,1]$ rather than $[-1,1]$. For this reason, we refer to these polynomials as half-range Chebyshev polynomials of the first kind. The orthogonal polynomials are guaranteed to exist, because the weight function is positive and integrable [10].

\subsubsection{Odd functions}

Next, we consider the odd part of $G_{n}$ and we denote by

$$
\mathcal{S}_{n}:=\operatorname{span} S_{n}
$$

the $n$-dimensional space spanned by the sine functions. The counterpart of (14) is the observation that

$$
\sin (k+1) \frac{\pi}{2} x=U_{k}\left(\cos \frac{\pi}{2} x\right) \sin \frac{\pi}{2} x
$$

We have the following theorem.

Theorem 3.4. Let $U_{k}^{h}(y)$ be the sequence of orthogonal polynomials satisfying

$$
\frac{4}{\pi} \int_{0}^{1} U_{k}^{h}(y) y^{l} \sqrt{1-y^{2}} \mathrm{~d} y=\delta_{k-l}, \quad l=0, \ldots, k-1 .
$$

Then the set $\left\{T_{k}^{h}\left(\cos \frac{\pi}{2} x\right) \sin \frac{\pi}{2} x\right\}_{k=0}^{n-1}$ is an orthonormal basis for $\mathcal{S}_{n}$ on $[-1,1]$. 
Proof. Consider a function $g \in \mathcal{S}_{n}$. Since $g$ is odd, the function $g(x) / \sin \frac{\pi}{2} x$ is well-defined on $[-1,1]$ and it is even. We again restrict ourselves to the interval $[0,1]$ and perform the substitution $y=\cos \frac{\pi}{2} x$. It follows from the Chebyshev polynomial property (16) that

$$
\frac{g\left(\frac{2}{\pi} \cos ^{-1} y\right)}{\sin \frac{\pi}{2} \frac{2}{\pi} \cos ^{-1} y}=\frac{g\left(\frac{2}{\pi} \cos ^{-1} y\right)}{\sqrt{1-y^{2}}}
$$

is a polynomial in $y$ on $[0,1]$, which we denote by $Q_{g}(y)$.

Conversely, from (16) it also follows that each polynomial $q(y)$ of degree less than $n$ corresponds to a function $q\left(\cos \frac{\pi}{2} x\right) \sin \frac{\pi}{2} x \in \mathcal{S}_{n}$, because the set of Chebyshev polynomials of the second kind up to degree $n-1$ is a basis for the space of all polynomials up to degree $n-1$.

We enforce orthogonality of $g$ to the function $\tilde{g} \in \mathcal{S}_{n-1}$. This means that

$$
\begin{aligned}
\int_{-1}^{1} g(x) \tilde{g}(x) \mathrm{d} x & =2 \int_{0}^{1} g(x) \tilde{g}(x) \mathrm{d} x \\
& =2 \int_{1}^{0} \frac{g\left(\frac{2}{\pi} \cos ^{-1} y\right)}{\sqrt{1-y^{2}}} \frac{\tilde{g}\left(\frac{2}{\pi} \cos ^{-1} y\right)}{\sqrt{1-y^{2}}}\left(1-y^{2}\right) \frac{-2}{\pi} \frac{1}{\sqrt{1-y^{2}}} \mathrm{~d} y \\
& =\frac{4}{\pi} \int_{0}^{1} Q_{g}(y) Q_{\tilde{g}}(y) \sqrt{1-y^{2}} \mathrm{~d} y \\
& =0 .
\end{aligned}
$$

If $g(x)=U_{n-1}^{h}\left(\cos \frac{\pi}{2} x\right) \sin \frac{\pi}{2} x$, then $Q_{g}(y)=U_{n-1}^{h}(y)$ and $g(x)$ is orthogonal to all functions in $\mathcal{S}_{n-1}$. The normalization implicit in (17) corresponds exactly to the normalization of $U_{k}^{h}\left(\cos \frac{\pi}{2} x\right) \sin \frac{\pi}{2} x$ in $L_{[-1,1]}^{2}$. This proves the result.

Similar to the case of even functions, the orthogonal polynomials $U_{n}^{h}(y)$ have the same weight function as the Chebyshev polynomials of the second kind, but they are defined on half the range. We denote them by half-range Chebyshev polynomials of the second kind.

\subsubsection{Properties of the orthogonal polynomials}

Fig. 2 shows the first few half-range Chebyshev polynomials of the first and of the second kind. They are not classical polynomials. The polynomials of the first kind, shown in the left panel of Fig. 2, are almost equi-oscillatory except near the origin. This is reminiscent of classical Chebyshev polynomials of the first kind. Their value at $y=1$ converges rapidly to $2^{-1 / 4} \approx 0.84 \ldots$, but they are unbounded at $y=0$. The half-range Chebyshev polynomials of the second kind are unbounded in both endpoints.

The growth of the polynomials in $[-1,1]$ follows from classical results in the theory of orthogonal polynomials. We have the following results.

Theorem 3.5. The half-range Chebyshev polynomials of the first and second kinds satisfy

$$
T_{k}^{h}(0) \sim \sqrt{k}, \quad T_{k}^{h}(1) \sim 1, \quad U_{k}^{h}(0) \sim \sqrt{k}, \quad U_{k}^{h}(1) \sim k
$$

for $k \rightarrow \infty$. 

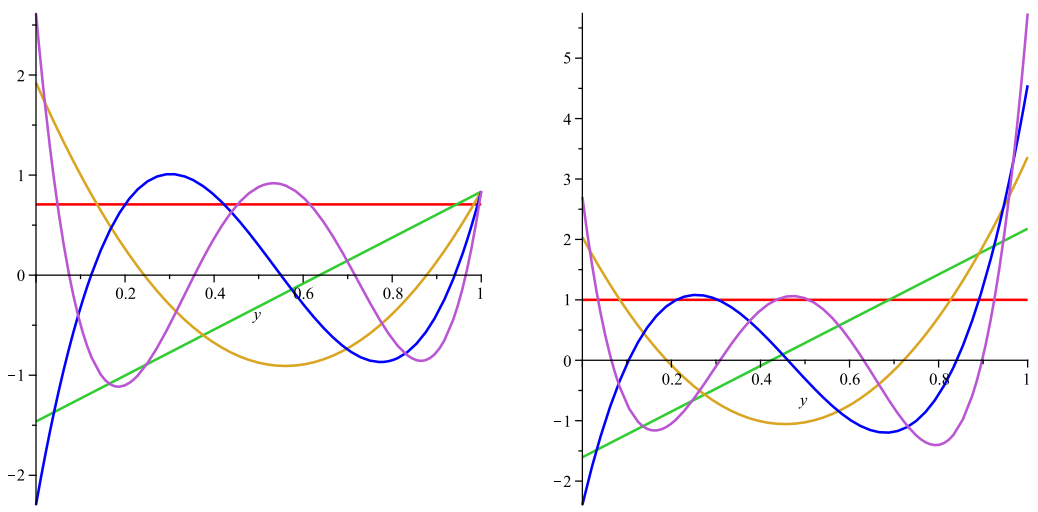

Figure 2: Plots of the half-range Chebyshev polynomials of the first kind (left panel) and of the second kind (right panel).

This follows from the work of Bernstein [1], who studied polynomials orthonormal on $[-1,1]$ with respect to a weight function of the form $w(x)=$ $w_{0}(x)(1-x)^{\alpha}(1+x)^{\beta}$.

\subsection{The exact solution}

With an orthonormal basis of our search space in hand, the exact solution to Problem 1.1 readily follows from the orthogonal projection. This shows existence and uniqueness of the solution for any given $f \in L_{[-1,1]}^{2}$.

Theorem 3.6. For a given $f \in L_{[-1,1]}^{2}$, the solution to Problem 1.1 is

$$
g_{n}(x)=\sum_{k=0}^{n} a_{k} T_{k}^{h}\left(\cos \frac{\pi}{2} x\right)+\sum_{k=0}^{n-1} b_{k} U_{k}^{h}\left(\cos \frac{\pi}{2} x\right) \sin \frac{\pi}{2} x
$$

where

$$
a_{k}=\int_{-1}^{1} f(x) T_{k}^{h}\left(\cos \frac{\pi}{2} x\right) \mathrm{d} x
$$

and

$$
b_{k}=\int_{-1}^{1} f(x) U_{k}^{h}\left(\cos \frac{\pi}{2} x\right) \sin \frac{\pi}{2} x \mathrm{~d} x .
$$

Proof. The result is the orthogonal projection onto the orthonormal bases that were found in Theorem 3.3 and Theorem 3.4.

The coefficients can also be found from polynomial expansions after the transformation $y=\cos \frac{\pi}{2} x$, which leads to more standard expressions, but one has to distinguish between the even and the odd parts of $f$.

Corollary 3.7. For a given $f \in L_{[-1,1]}^{2}$, denote by $f_{e}$ and $f_{o}$ its even and odd parts,

$$
f_{e}(x)=\frac{f(x)+f(-x)}{2} \quad \text { and } \quad f_{o}(x)=\frac{f(x)-f(-x)}{2} .
$$


The solution to Problem 1.1 is given by (19) with

$$
a_{k}=\frac{4}{\pi} \int_{0}^{1} f_{e}\left(\frac{2}{\pi} \cos ^{-1} y\right) T_{k}^{h}(y) \frac{1}{\sqrt{1-y^{2}}} \mathrm{~d} y
$$

and

$$
b_{k}=\frac{4}{\pi} \int_{0}^{1} f_{o}\left(\frac{2}{\pi} \cos ^{-1} y\right) U_{k}^{h}(y) \mathrm{d} y .
$$

Proof. Write $f(x)=f_{e}(x)+f_{o}(x)$ in the expressions for $a_{k}$ and $b_{k}$ in Theorem 3.6. The odd part of $f$ is irrelevant for $a_{k}$, since $T_{k}^{h}\left(\cos \frac{\pi}{2} x\right)$ is even. Likewise, the even part of $f$ is irrelevant for $b_{k}$. The result follows immediately from the substitution $y=\cos \frac{\pi}{2} x$. (Note, in the case of $b_{k}$, that the Jacobian of the transformation partially cancels with the additional sine function in (21).)

Let us for convenience define the functions

$$
f_{1}(y):=f_{e}\left(\frac{2}{\pi} \cos ^{-1} y\right)=f_{e}(x),
$$

and

$$
f_{2}(y):=\frac{f_{o}\left(\frac{2}{\pi} \cos ^{-1} y\right)}{\sqrt{1-y^{2}}}=\frac{f_{o}(x)}{\sin \frac{\pi}{2} x},
$$

where $x=\frac{2}{\pi} \cos ^{-1} y$. Corollary 3.7 informs us that the exact solution to the Fourier extension problem is found from the least squares polynomial approximations of the functions $f_{1}(y)$ and $f_{2}(y)$ with respect to the weight functions $\frac{4}{\pi} \frac{1}{\sqrt{1-y^{2}}}$ and $\frac{4}{\pi} \sqrt{1-y^{2}}$ on the interval $[0,1]$.

\subsection{Convergence}

The explicit expressions for the exact solution of Problem 1.1 in terms of orthogonal polynomials enables using standard theory in polynomial approximation to derive the convergence rate of the approximation. We will focus in this paper only on the case of analytic $f$.

\subsubsection{Preparatory lemmas}

First, we examine the convergence of polynomial least squares approximations on the interval $[0,1]$. Let $p_{n}(y)$ be a sequence of polynomials, orthonormal with respect to the positive and integrable weight function $w(y)$ on $[0,1]$. The weighted least squares polynomial approximation to a function $h$ is given by

$$
h_{n}(y)=\sum_{k=0}^{n} c_{n} p_{n}(y)
$$

with

$$
c_{n}=\int_{0}^{1} f(y) p_{n}(y) w(y) \mathrm{d} y .
$$

The convergence rate of this approximation is similar to that in Theorem 2.1. In the following lemma, we denote by $L_{[0,1], w}^{2}$ the weighted $L^{2}$-space with norm

$$
\|f\|_{L_{[0,1], w}^{2}}=\sqrt{\int_{0}^{1} f(x)^{2} w(x) \mathrm{d} x} .
$$


Lemma 3.8. Let $h$ be analytic with $|h(z)| \leq M$ in the region bounded by the ellipse with foci 0 and 1 and major and minor semiaxis lengths summing to $\rho / 2$. If $\rho>1$, then

$$
\left\|h-h_{n}\right\|_{L_{[0,1], w}^{2}} \sim \rho^{-n}, \quad n \rightarrow \infty .
$$

Proof. The substitution $u=2 z-1$ maps an ellipse with foci 0 and 1 and major and minor semiaxis lengths summing to $\rho / 2$ to an ellipse with foci \pm 1 and major and minor semiaxis lengths summing to $\rho$. Hence, if $\rho>1$, then from the theory of Chebyshev expansions in $\S 2.1$ a polynomial $q$ of degree $n$ exists such that

$$
h(z)-q(z) \sim \rho^{-n}, \quad z \in[0,1] .
$$

Since $h_{n}$ is the weighted least squares approximation to $h$, we have

$$
\begin{aligned}
\int_{0}^{1}\left(h(z)-h_{n}(z)\right)^{2} w(z) \mathrm{d} z & \leq \int_{0}^{1}(h(z)-q(z))^{2} w(z) \mathrm{d} z \\
& \leq\|h-q\|_{\infty}\|w\|_{1} .
\end{aligned}
$$

Since $w$ is positive and integrable, this proves the result.

We will also make use of the following result later on.

Lemma 3.9. Let $f$ be even and analytic. Then $f$ is periodic on $[-T, T]$ if and only if $f$ is even with respect to $T$.

Proof. Assume first that $f$ is even and $2 T$-periodic. Then we have

$$
f(T-a)=f(-T+a)=f(T+a),
$$

so $f$ is even around $T$. Next, if $f$ is even around 0 and $T$, then we have

$$
f(a)=f(T-(T-a))=f(T+(T-a))=f(2 T-a)=f(a-2 T),
$$

so $f$ is $2 T$-periodic.

The proof of the following lemma is analogous and is omitted.

Lemma 3.10. Let $f$ be odd and analytic. Then $f$ is periodic on $[-T, T]$ if and only if $f$ is odd with respect to $T$.

\subsubsection{Convergence}

In order to understand the convergence rate of the solution to Problem 1.1 for analytic $f$, we should know the domain of analyticity of the functions $f_{1}(y)$ and $f_{2}(y)$. It turns out that the substitution $x=\frac{2}{\pi} \cos ^{-1} y$ introduces singularities in the complex plane such that, even when $f$ is entire, the convergence rate of the Fourier extension problem is bounded in most cases.

Definition 3.11. The Fourier extension constant is

$$
E=3+2 \sqrt{2} .
$$




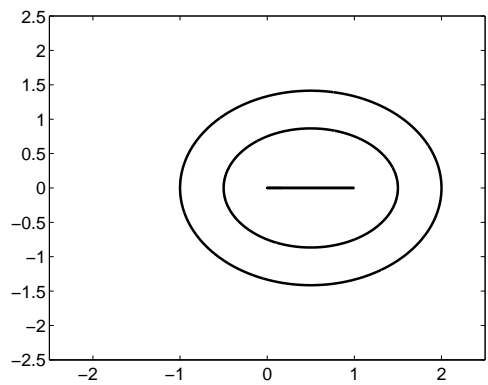

(a) Ellipses with foci 0 and 1 and major semiaxis length $R$

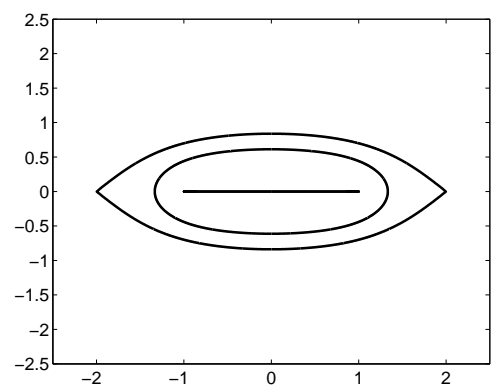

(b) The corresponding domains $D(R)$

Figure 3: The domains of analyticity for $\tilde{f}(y)$ (left panel) for $R=1 / 2,1,3 / 2$ and the corresponding domains of analyticity $D(R)$ for $f(x)$ (right panel).

Lemma 3.12. Let $f(x)$ be even and analytic in a neighbourhood of $[-1,1]$ and define

$$
\tilde{f}(y)=f\left(\frac{2}{\pi} \cos ^{-1} y\right)
$$

If $\tilde{f}(y)$ is analytic in the region bounded by an ellipse with foci 0 and 1 and major and minor semiaxis lengths summing to $\rho / 2$, then

$$
\rho \leq E
$$

unless $f$ is analytic and periodic on $[-2,2]$.

Proof. The major semiaxis length $a$, minor semiaxis length $b$ and distance $2 c$ between the two foci of an ellipse are related by $a^{2}-b^{2}=c^{2}$. For an ellipse with foci 0 and 1 , this means $a^{2}-b^{2}=1 / 4$.

The function $\cos ^{-1} y$ has square root type singularities at $y_{1}=1$ and $y_{2}=$ -1 . However, since $\frac{2}{\pi} \cos ^{-1} y_{1}=0$ and since $f$ is assumed to be even, the singularity squares away and $\tilde{f}$ is analytic at $y=1$.

A possible singularity remains at $y_{2}$. Assume sufficient analyticity of $f$, in order to get an upper bound for $\rho$. If $\tilde{f}$ is also analytic at $y_{2}$, then $f$ is necessarily even around $\frac{2}{\pi} \cos ^{-1} y_{2}=2$. Since $f$ is then even around 0 and 2 , it follows from Lemma 3.9 that $f$ is periodic on $[-2,2]$.

Otherwise, in the general case where $f$ is not periodic on $[-2,2]$, the major semiaxis length of an ellipse with foci 0 and 1 bounding the region of analyticity of $\tilde{f}$ is limited to $a=\frac{3}{2}$, i.e., to the distance between the center of the ellipse at $y=1 / 2$ and the nearest singularity at $y=-1$. The sum of major and minor semiaxis lengths is $a+\sqrt{a^{2}-1 / 4}=\rho / 2$, which for $a=3 / 2$ leads to the bound for $\rho$.

The (open) domain of analyticity of $\tilde{f}(y)$ should be an ellipse with foci 0 and 1 . This domain is mapped by

$$
x=\frac{2}{\pi} \cos ^{-1} y
$$


to the corresponding domain of analyticity of the even function $f$. The form of this domain is shown in the right panel of Figure 3. We denote the latter by $D(R)$, where $R$ is the major semiaxis length of the ellipse. Note that the intersection of $D(3 / 2)$ with the real axis is the interval $[-2,2]$.

Theorem 3.13. If $f$ is analytic in the domain $D(R)$, with $R>1 / 2$, then the solution $g_{n}$ to Problem 1.1 satisfies

$$
\left\|f-g_{n}\right\| \sim \rho^{-n}
$$

with

$$
\rho=\min \left(E, 2 R+\sqrt{4 R^{2}-1}\right),
$$

unless $f$ is analytic and periodic on $[-2,2]$.

Proof. First, we can invoke Lemma 3.12 for the functions $f_{e}(x)$ and $f_{o}(x) / \sin \frac{\pi}{2} x$, which are both even. The function $f_{e}(x)$ is analytic in $D(R)$ by construction. The function $f_{o}(x) / \sin \frac{\pi}{2} x$ may have poles at $x= \pm 2 n$ for $n \in \mathbb{N}_{0}$. If $f_{o}(x)$ is periodic on $[-2,2]$, then it follows from Lemma 3.10 that it vanishes at all possible poles, so $f_{o}(x) / \sin \frac{\pi}{2} x$ is analytic in $D(r)$. Otherwise, if $f_{o}(x)$ is not periodic, $f_{o}(x) / \sin \frac{\pi}{2} x$ is analytic only in

$$
D:=D(R) \cap D(3 / 2) .
$$

Next, recall the definitions (25) and (26) of $f_{1}$ and $f_{2}$. Denote by $p_{n}(y)$ the polynomial least squares approximation of degree $n$ of $f_{1}(y)$ with respect to the weight function $\frac{4}{\pi} \frac{1}{\sqrt{1-y^{2}}}$, and by $q_{n}(y)$ the polynomial least squares approximation of degree $n-1$ of $f_{2}(y)$ with respect to the weight function $\frac{4}{\pi} \sqrt{1-y^{2}}$. Note that, since odd and even functions are orthogonal on $[-1,1]$, we have

$$
\left\|f-g_{n}\right\|^{2}=\left\|f_{e}-p_{n}\left(\cos \frac{\pi}{2} \cdot\right)\right\|^{2}+\left\|f_{o}-q_{n}\left(\cos \frac{\pi}{2} \cdot\right) \sin \left(\frac{\pi}{2} \cdot\right)\right\|^{2} .
$$

Define $\rho$ as in (29). From Theorem 2.1, we find that

$$
\frac{4}{\pi} \int_{0}^{1}\left(f_{1}(y)-p_{n}(y)\right)^{2} \frac{1}{\sqrt{1-y^{2}}}(y) \mathrm{d} y \sim \rho^{-2 n},
$$

and

$$
\frac{4}{\pi} \int_{0}^{1}\left(f_{2}(y)-q_{n}(y)\right)^{2} \sqrt{1-y^{2}}(y) \mathrm{d} y \sim \rho^{-2(n-1)} \sim \rho^{-2 n} .
$$

Finally, we note that the two integrals in the expressions above correspond exactly to the two norms in the right hand side of (30) by letting $y=\cos \frac{\pi}{2} x$. This proves the result.

Corollary 3.14. Under the conditions of Theorem 3.13, the coefficients $a_{k}$ and $b_{k}$ of $g_{n}$ in the form of (19) satisfy $a_{k}, b_{k} \sim \rho^{-n}$.

Figure 4 shows the size of the $a_{k}$ and $b_{k}$ coefficients of $g_{n}$ in the form (19) for two functions $f$. The first function (left panel) is entire but not periodic. The convergence rate is $E^{-n}$, as predicted by the theory. The second function (right panel) is entire and periodic. The convergence rate for this example is 


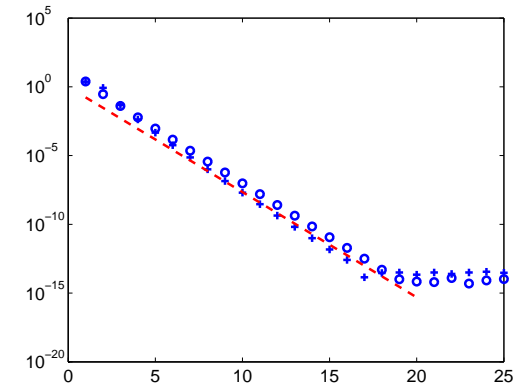

(a) $f(x)=2 x^{2}+3 x+1$

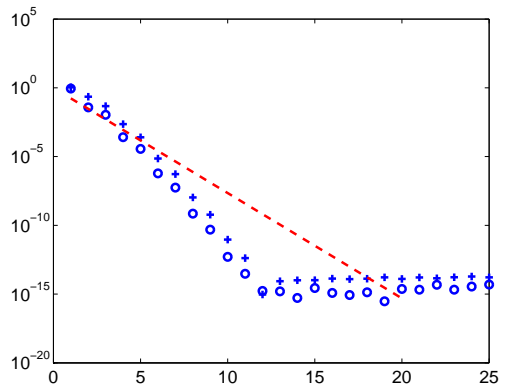

(b) $f(x)=\cos \cos \frac{\pi}{2} x+\sin \sin \frac{\pi}{2} x$

Figure 4: Logarithmic plots of the $a_{k}$ coefficients $(+)$ and $b_{k}$ coefficients (o) of $g_{n}$ in the form (19). Also shown is the curve $E^{-n}$ (striped line).

faster than exponential. The computations were performed in Matlab in double precision using the algorithm outlined in $\S 5$.

We conclude in this section that the convergence rate is truly exponential of the form $\mathrm{e}^{-c n}$, though the exponent is limited in most cases to $\log E \approx 1.76$. It is not surprising that functions periodic on $[-2,2]$ are an exception to this rule. Indeed, such functions have rapidly converging classical Fourier series on $[-2,2]$ which, depending on nearby singularities in the complex plane, may converge faster. Note however that $g_{n}(x)$ does not necessarily coincide with the classical Fourier series of $f$ on $[-2,2]$, truncated after $2 n+1$ terms.

\subsection{The Fourier extension on $[-2,2]$}

In this section, we analyze the behavior of the Fourier extension of $f$ outside the interval $[-1,1]$. To that end, we have to examine the rate of growth of the orthogonal polynomials in $[-1,1]$.

Theorem 3.15. The polynomials $T_{k}^{h}(y)$ satisfy

$$
\left(\left\|T_{k}^{h}\right\|_{L_{[-1,1]}^{\infty}}\right)^{-1}=o\left(\rho^{-k}\right), \quad \forall 0<\rho<E .
$$

Proof. Assume an even function $f$ that is analytic in $D(3 / 2)$ but with a singularity at $x=2$, i.e., $f$ is not analytic in $\overline{D(3 / 2)}$. This implies by Corollary 3.14 that $a_{k} \sim E^{-k}$ (note that $b_{k}=0$ for an even function).

Now assume that the maximum of the polynomials $T_{k}^{h}$ on the interval $[-1,1]$ grows only as fast as $\rho^{k}$ with $\rho<E$. Then the series (19) converges exponentially for $x \in[-2,2]$, since the terms

$$
a_{k} T_{k}^{h}\left(\cos \frac{\pi}{2} x\right) \sim(E-\rho)^{-k}
$$

decay exponentially fast. This means that $g_{n}$ converges to a fixed function $g$ with an exponentially decaying Fourier series. The exponential decay implies that $g$ is analytic on $[-2,2][21]$. But $g_{n}$ converges to $f$ on $[-1,1]$ which is not analytic on $[-2,2]$. We arrive at a contradiction, so the polynomials must grow faster than $\rho^{k}$. 


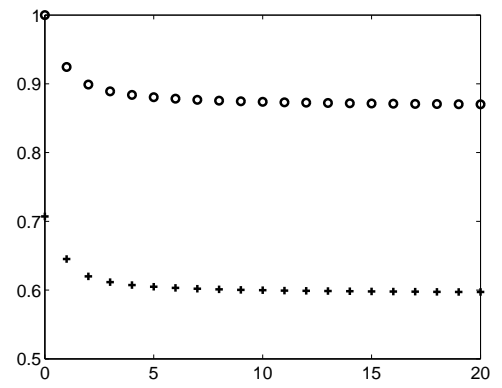

Figure 5: Plots of the ratios $\frac{T_{k}^{h}(-1)}{E^{k}}(+)$ and $\frac{U_{k}^{h}(-1)}{E^{k}}(\mathrm{o})$.

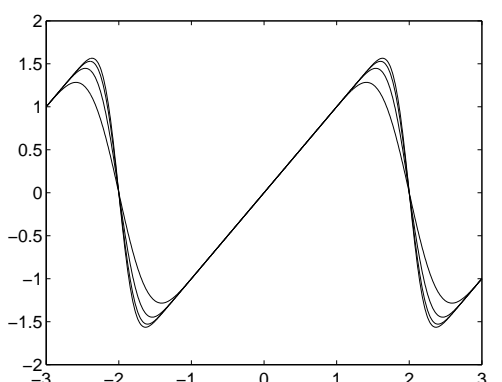

(a) $g_{n}(x)$

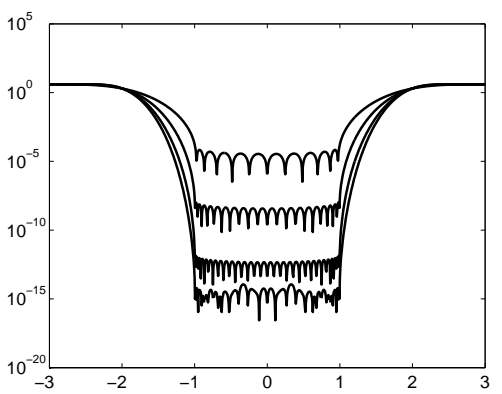

(b) $\left|x-g_{n}(x)\right|$

Figure 6: Illustration of the Fourier extension $g_{n}(x)$ of $f(x)=x$ for $n=$ $5,10,15,20$. Slow growth outside $[-1,1]$ is seen in the left panel. The right panel shows that the approximation is very accurate on $[-1,1]$ including in the endpoints.

Theorem 3.15 says that the polynomials grow faster than $\rho^{k}$ for any $\rho<E$. A similar statement can be made regarding the polynomials $U_{k}^{h}(y)$. The result of this theorem is not entirely sharp however. Numerical experiments indicate that

$$
\left\|T_{k}^{h}\right\|_{L_{[-1,1]}^{\infty}} \sim E^{k}, \quad k \rightarrow \infty .
$$

Since the polynomials grow monotonically outside the interval [0,1], they attain their maximum on $[-1,1]$ in the left endpoint $y=-1$. Figure 5 illustrates that the ratios $\frac{T_{k}^{h}(-1)}{E^{k}}$ and $\frac{U_{k}^{h}(-1)}{E^{k}}$ indeed quickly converge to a limit.

This growth of the polynomials compensates the rapid decay of the expansion coefficients $a_{k}$ and $b_{k}$, such that $g_{n}$ may actually diverge outside $[-1,1]$. We illustrate this for the function $f(x)=x$ in Figure 6. The Fourier extension grows slowly outside the interval $[-1,1]$. The approximation on $[-1,1]$ is very accurate however, up to and including in the endpoints.

The growth outside $[-1,1]$ is more pronounced for the function $f(x)=$ $1 /\left(1+2 x^{2}\right)$, which has two poles $\pm \frac{i}{\sqrt{2}}$ near the real axis. This is shown in Figure 7 . Note that the approximation remains very accurate on $[-1,1]$, roughly 


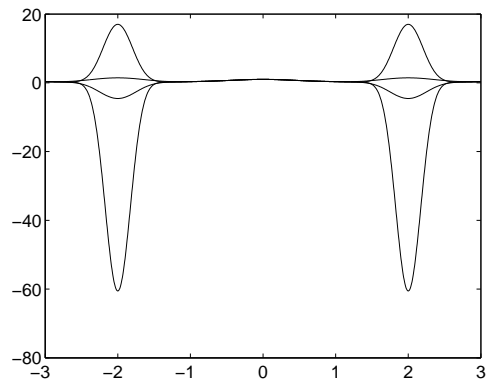

(a) $g_{n}(x)$

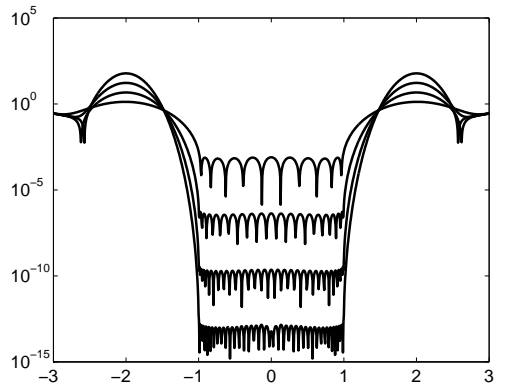

(b) $\left|x-g_{n}(x)\right|$

Figure 7: Same as Figure 6 but for the function $f(x)=\frac{1}{1+2 x^{2}}$. The growth of the Fourier extension outside $[-1,1]$ is more pronounced in this example, but the approximation remains very accurate on $[-1,1]$.

achieving machine precision.

\section{Numerical least squares methods}

There are many approximate representations of $f$ in the space $G_{n}$. In this section, we describe the mechanism that makes well designed numerical methods favour accurate representations with small Fourier coefficients over the exact solution to Problem 1.1 that yields large Fourier coefficients.

\subsection{Projection methods and collocation methods}

For simplicity of notation in this section, we will focus on solving Problem 1.1 in the function space $G_{2 n}$ rather than the space $G_{n}$.

A straightforward translation of Problem 1.1 into a linear algebra problem is the following. Given a basis $\left\{\phi_{j}\right\}_{j=1}^{4 n+1}$ of $G_{2 n}$, the solution to Problem 1.1 is

$$
g_{n}(x)=\sum_{j=1}^{4 n+1} x_{j} \phi_{j}(x),
$$

where the coefficients $x_{j}$ are found by solving the linear system of equations

$$
A x=B .
$$

The entries of the matrix $A \in \mathbb{R}^{(4 n+1) \times(4 n+1)}$ are given by

$$
A_{i, j}=<\phi_{j}, \phi_{i}>\text {, }
$$

and the elements of $B \in \mathbb{R}^{4 n+1}$ are

$$
B_{i}=<f, \phi_{i}>.
$$

Note that by $\left\langle\cdot, \cdot>\right.$ we mean the inner product on $L_{[-1,1]}^{2}$. In exact arithmetic, this projection method produces the exact solution to Problem 1.1, regardless of the basis used. 
Alternatively, one may use a collocation approach. For a set $\left\{y_{j}\right\}_{j=1}^{M}$ of collocation points, with $y_{j} \in[-1,1]$ and $M \geq 4 n+1$, solve the linear system of equations

$$
\tilde{A} x=\tilde{B}
$$

where $\tilde{A}_{i j}=\phi_{j}\left(y_{i}\right)$ and $\tilde{B}_{i}=f\left(y_{i}\right)$

These methods were used in $[3,5]$ to solve the Fourier extension problem numerically. Note that

$$
A \approx c(M) \tilde{A}^{T} \tilde{A},
$$

i.e., the linear system $A x=B$ approximates the normal equations of the least squares problem (34), up to a constant $c(M)$ that depends on $M$. For $M$ equidistant points on $[-1,1]$, one can verify that $c(M)=\frac{2}{M-1} \cdot{ }^{3}$ Expression (35) implies that

$$
\kappa(A) \approx \kappa(\tilde{A})^{2} .
$$

One may in general expect better numerical results from the collocation approach [19].

\subsection{Block structure and other properties of $A$}

We examine the structure and properties of the matrix $A$. We start with the following observation.

Lemma 4.1. The matrix $A$ is symmetric and positive definite.

Proof. It follows from the theory in $\S 3$ that $D_{2 n}$ is a basis for $G_{2 n}$ on $[-1,1]$. Since $A$ consists of all inner products of the basis functions, it is symmetric and positive definite.

The choice $T=2$ leads to a matrix $A$ with a simple structure. Recall from Lemma 3.2 that in this case the set $D_{2 n}$, which spans $G_{2 n}$, consists of Laplace eigenfunctions subject to Neumann or Dirichlet boundary conditions. We have

$$
D_{2 n}=L^{N} \cup L^{D}
$$

where (note that we redefine $L^{N}$ and $L^{D}$ here to be finite sets)

$$
L^{N}:=\left\{\phi_{j}^{N}\right\}_{j=1}^{2 n+1}=\left\{\frac{1}{\sqrt{2}}\right\} \cup\{\cos \pi k x\}_{k=1}^{n} \cup\left\{\sin \pi\left(k+\frac{1}{2}\right) x\right\}_{k=0}^{n-1},
$$

and

$$
L^{D}:=\left\{\phi_{j}^{D}\right\}_{j=1}^{2 n}=\left\{\cos \pi\left(k+\frac{1}{2}\right) x\right\}_{k=0}^{n-1} \cup\{\sin \pi k x\}_{k=1}^{n} .
$$

With that order of the basis functions, the matrix $A$ has the following block structure:

$$
A=\left[\begin{array}{cc}
I_{2 n+1} & C \\
C^{T} & I_{2 n}
\end{array}\right]
$$

where $I_{m} \in \mathbb{R}^{m \times m}$ is the identity matrix. The matrix $C \in \mathbb{R}^{(2 n+1) \times 2 n}$ is a coupling matrix given by

$$
C_{i, j}=<\phi_{j}^{D}, \phi_{i}^{N}>
$$

It turns out that all eigenvalues and eigenvectors of $A$ are easily determined.

\footnotetext{
${ }^{3}$ This follows from the observation that each element of $\tilde{A}^{T} \tilde{A}$ is a low order composite quadrature approximation to the integrals of the form (33) with step size $h=2 /(M-1)$.
} 


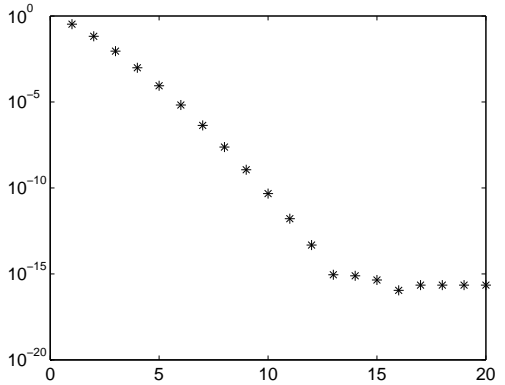

(a) $1-\sigma_{k}$

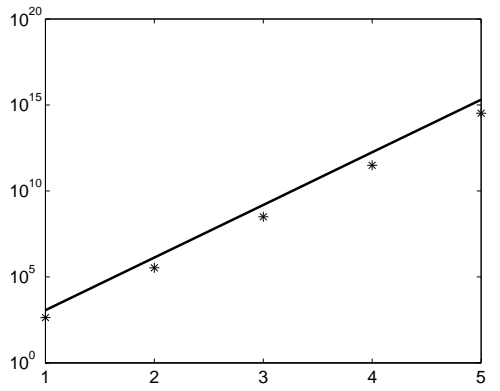

(b) $\kappa(A)$ and $E^{4 n}$

Figure 8: The distribution of the singular values of $C$ for $n=10$ (left panel) and the condition number of $A$ versus $E^{4 n}$ (right panel).

Theorem 4.2. Let $C=U \Sigma V^{T}$ be the singular value decomposition of $C$. For each singular value $\sigma_{k}$, with left and right singular vectors $u_{k}$ and $v_{k}$, we have

$$
A\left[\begin{array}{l}
u_{k} \\
v_{k}
\end{array}\right]=\left(1+\sigma_{k}\right)\left[\begin{array}{l}
u_{k} \\
v_{k}
\end{array}\right],
$$

and

$$
A\left[\begin{array}{c}
u_{k} \\
-v_{k}
\end{array}\right]=\left(1-\sigma_{k}\right)\left[\begin{array}{c}
u_{k} \\
-v_{k}
\end{array}\right] \text {. }
$$

We also have

$$
A\left[\begin{array}{c}
u_{2 n+1} \\
0
\end{array}\right]=\left[\begin{array}{c}
u_{2 n+1} \\
0
\end{array}\right] .
$$

Proof. Consider the first eigenvalue $\left(1+\sigma_{k}\right)$. Since $C v_{k}=\sigma_{k} u_{k}$ and $C^{T} u_{k}=$ $\sigma_{k} v_{k}$, we have

$$
A\left[\begin{array}{l}
u_{k} \\
v_{k}
\end{array}\right]=\left[\begin{array}{c}
u_{k}+C v_{k} \\
C^{T} u_{k}+v_{k}
\end{array}\right]=\left(1+\sigma_{k}\right)\left[\begin{array}{c}
u_{k} \\
v_{k}
\end{array}\right] .
$$

The case of the eigenvalue $\left(1-\sigma_{k}\right)$ is analogous. Together, they account for $4 n$ of the $4 n+1$ eigenvalues of $A$.

Since $C$ is not square, there are $2 n+1$ left singular vectors $u_{k}$ but only $2 n$ right singular vectors $v_{k}$. Because $u_{2 n+1}$ lies in the null space of $C^{T}$, we have $C^{T} u_{2 n+1}=0$ and the same reasoning as above yields the last eigenvalue 1 with the given corresponding eigenvector.

Corollary 4.3. $0 \leq \sigma_{k}<1$.

Proof. Singular values are positive by construction. Since $A$ is positive definite, all its eigenvalues are positive. This means that $1-\sigma_{k}>0$ or $\sigma_{k}<1$.

Corollary 4.4. $0<\lambda_{k}<2$.

The eigenvalues of $A$ are bounded by 0 and 2. Numerical experiments indicate that the eigenvalues rapidly accumulate near 0 and 2. Figure 8 illustrates 
the accumulation of the $2 n$ singular values $\sigma_{k}$ near 1 for $n=10$. The eigenvalues of $A$ are similarly distributed near 0 and 2. This means that $\|A\|$ is bounded, but $\left\|A^{-1}\right\|$ is not. Numerical experiments suggest that

$$
\kappa(A) \sim E^{4 n}, \quad n \rightarrow \infty
$$

The condition number of $A$ reaches $1 e 15$ for $n=5$. This corresponds to a matrix of size $4 n+1=21$ by 21 .

Remark 4.5. Let us briefly comment on the meaning of the eigenvectors $\left[\begin{array}{l}u_{k} \\ v_{k}\end{array}\right]$. The fact that $\sigma_{k} \approx 1$ means that the spaces $\mathcal{L}^{N}:=\operatorname{span} L^{N}$ and $\mathcal{L}^{D}:=\operatorname{span} L^{D}$ are, in a sense, similar. We have $\sum_{k} u_{k} \phi_{k}^{N} \approx \sum_{k} v_{k} \phi_{k}^{D}$. Any function giving rise to the right hand side vector $B=\left[\begin{array}{l}u_{k} \\ v_{k}\end{array}\right]$ projects similarly onto the spaces $\mathcal{L}^{N}$ and $\mathcal{L}^{D}$. The eigenvalue $1+\sigma_{k}$ is bounded away from zero and the corresponding solution to $A x=B$ is therefore well behaved. In contrast, any function that leads to $B=\left[\begin{array}{c}u_{k} \\ -v_{k}\end{array}\right]$ projects differently onto these spaces - the projections are nearly equal but opposite in sign. The corresponding solution vector $x$ is large, because the eigenvalue $1-\sigma_{k}$ is small. This case is increasingly unlikely, since the projections of a function onto $\mathcal{L}^{N}$ and onto $\mathcal{L}^{D}$ both converge to the function itself. It may happen however if $n$ is not large enough. We will illustrate further on the observation that accurate representations of $f$ with small Fourier coefficients can only be found provided $n$ is sufficiently large.

\subsection{Stability}

Assume we are given a bounded function $f \in L_{[-1,1]}^{2}$. Since our basis is the truncated concatenation of two orthonormal bases for $L_{[-1,1]}^{2}$, we find based on Parseval's identity that

$$
\|B\|^{2} \leq\|f\|^{2}+\|f\|^{2}=2\|f\|^{2} .
$$

Bounded $f$ gives rise to a bounded right hand side of (32). Ideally, we would like to obtain estimates

$$
c_{1}\|f\| \leq\|x\| \leq c_{2}\|f\| .
$$

However, such estimates obviously cannot hold with bounded constants, because the inverse of $A$ is unbounded. Based on Theorem 4.2 however, we can formulate a result that comes close.

Theorem 4.6. If $B \in \operatorname{span}\left\{\left[\begin{array}{l}u_{k} \\ v_{k}\end{array}\right]\right\}$, then

$$
\frac{1}{2}\|B\| \leq\|x\| \leq\|B\| .
$$

Proof. The right hand side of $A x=B$ lies in the space spanned by eigenvectors of $A$ with eigenvalues $1 \leq \lambda_{k}=1+\sigma_{k}<2$.

This result is encouraging, yet not very informative, since the singular vectors of $C$ are unknown and the condition on $B$ seems to be rather restrictive. 
The lower bound however has a precise meaning. Note that this lower bound actually holds for all $B$, since all eigenvalues are bounded by 2 . Because $\|B\| \rightarrow$ $\sqrt{2}\|f\|$, the bound converges to

$$
\frac{1}{\sqrt{2}}\|f\| \leq\|x\|
$$

This inequality corresponds exactly to inequality (8) in the theory of frames. Recall that this inequality expresses the fact that the energy of the coefficients of a representation of $f$ in a frame $\left\{\phi_{j}\right\}$ is minimized by taking inner products with the dual frame $\left\{\phi_{j}^{*}\right\}$. Thus, the lower bound (39) is achieved if

$$
x_{j}=<f, \phi_{j}^{*}>=\frac{1}{2}<f, \phi_{j}>,
$$

since for a tight frame the dual frame satisfies $\phi_{j}^{*}=\frac{1}{A} \phi_{j}$. (Note that the bound is reached in principle only when $n=\infty$.) The corresponding representation of $f$ is optimal in the sense of minimizing the energy of the coefficients, but it is not optimal in the sense of minimizing the norm $\|f-\cdot\|$. In fact, the series

$$
g^{*}:=\sum_{j=1}^{\infty} \frac{1}{2}<f, \phi_{j}>\phi_{j}
$$

converges rather slowly and exhibits the Gibbs phenomenon. ${ }^{4}$

\subsection{An underdetermined system of equations}

There are infinitely many representations of $f$ in the frame $D_{\infty}$. Therefore, if $n$ is sufficiently large, there are many approximate representations of $f$ in $D_{2 n}$. Two of these have received our special attention: one is the exact solution to Problem 1.1, another one is the dual frame representation (41). The former converges rapidly to $f$ but leads to unbounded Fourier coefficients. The latter converges slowly to $f$ but leads to bounded Fourier coefficients. One may expect a numerical method to yield a representation in between these two extremes. There is a well understood mechanism that makes certain numerical methods favour the latter.

We proceed formally. For an in-depth discussion of numerical methods for least squares problems, we refer the reader to [19]. Assume a numerical method that approximately solves an ill-conditioned system of equations by discarding small eigenvalues. Then system (32) can be formally replaced by

$$
A_{m} x=B
$$

where $A_{m}$ is a rank- $m$ approximation to $A$, with $m<4 n+1$. Assuming that $B$ lies in the range of $A_{m}$, the linear system of equations (42) is now underdetermined. There are many vectors $x$ that satisfy (42). However, exactly one solution $x^{*}$ of an underdetermined system minimizes $\|x\|$ among all possible solutions [19].

\footnotetext{
${ }^{4}$ The part corresponding to the Laplace-Neumann eigenfunctions converges pointwise in $[-1,1]$, the Laplace-Dirichlet eigenfunctions are responsible for the Gibbs phenomenon [16].
} 
For example, assume that $A=V \Lambda V^{T}$ is the eigenvalue decomposition of the symmetric and positive definite matrix $A$. Denote by $\tilde{\Lambda} \in \mathbb{R}^{m \times m}$ a truncation of $\Lambda$ that discards small eigenvalues, and let $\tilde{V} \in \mathbb{R}^{4 n+1 \times m}$ denote the corresponding eigenvectors. Then the underdetermined system of equations is

$$
\tilde{V} \tilde{\Lambda} \tilde{V}^{T} x=B .
$$

It is a property of the Fourier extension problem that $B$ lies approximately in the range of the low rank matrix, at least for sufficiently large $n$, as we will illustrate further below. The solution minimizing $\|x\|$ is found by solving the diagonal system

$$
\tilde{\Lambda} y=\tilde{V}^{T} B,
$$

and letting

$$
x^{*}=\tilde{V}^{T} y
$$

Note that the eigenvalue decomposition of $A$ can be computed efficiently from the singular value decomposition of $C$.

In the case of the collocation method, the approach is similar but based on a truncated singular value decomposition of $\tilde{A}$. Alternatively, results are shown further on by simply using the backslash operator in Matlab, $x=\tilde{A} \backslash \tilde{B}$. We only analyzed the eigenvalues of $A$. However, due to the property (35), the eigenvalues of $A$ are directly related to the singular values of $\tilde{A}$. This means that we may expect a similar energy-minimizing property of the solution as in the projection method. Results may even be better, because $\kappa(A) \sim E^{4 n}$ whereas $\kappa(\tilde{A}) \sim E^{2 n}$.

We conclude that the degrees of freedom that result from discarding small eigenvalues of $A$ or small singular values of $\tilde{A}$ can be used to minimize the norm of the solution. Hence, since the vector $x$ represents Fourier coefficients, unbounded Fourier coefficients can be avoided in practice. This is essentially due to numerical error: for large $n$ it is not feasible numerically to recover the exact solution of Problem 1.1.

\subsection{Numerical results}

We illustrate the statements made in this section with the example functions

$$
f_{1}(x)=x
$$

and

$$
f_{2}(x)=\mathrm{e}^{\sin (5.4 \pi x-2.7 \pi)-\cos (2 \pi x)} .
$$

The latter function was chosen in [5] as an example that leads to large Fourier coefficients for moderate $n$.

We use two numerical methods: the projection method, which corresponds to solving system (32), and the collocation method, which corresponds to solving system (34). In the former case, we compute the singular value decomposition of $C$, which yields the eigenvalue decomposition of $A$, and we compute the solution by (43). In the latter case, we compute the singular value decomposition of $\tilde{A}$. We chose $M=1000$ equidistant collocation points in $[-1,1]$. We used the threshold $1 e-12$ for the eigenvalues of $A$ and for the singular values of $\tilde{A}$. For both methods, we also compared results with the backslash-operator in Matlab. 


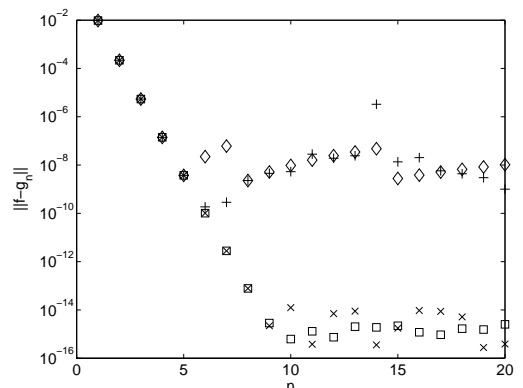

(a) $f 1$ : approximation error

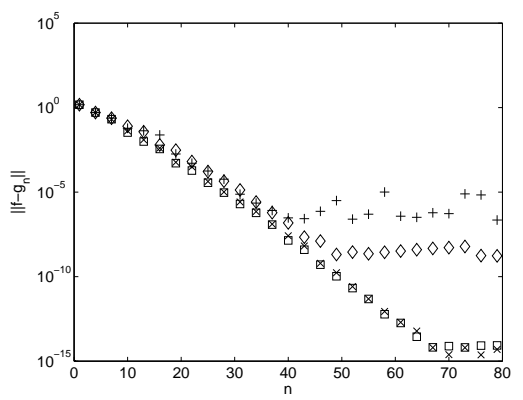

(c) f2: approximation error

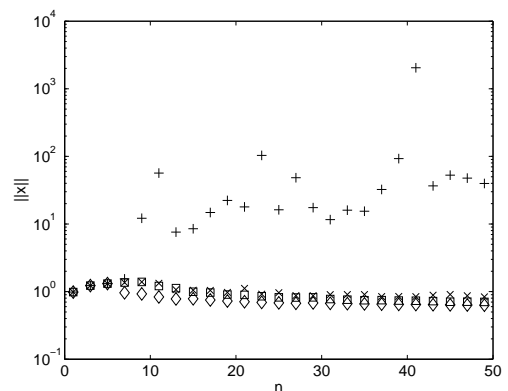

(b) $f 1$ : norm of solution vector

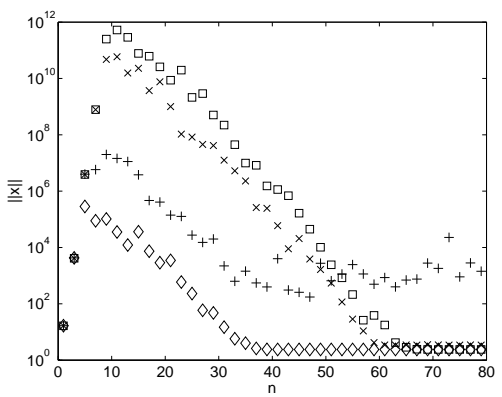

(d) $f 2$ : norm of solution vector

Figure 9: Convergence plot of four numerical methods: truncated eigenvalue decomposition of $A(\diamond)$, truncated singular value decomposition of $\tilde{A}(\square)$, Matlab solution $A \backslash B(+)$ and Matlab solution $\tilde{A} \backslash \tilde{B}(\times)$. The left panels show the approximation error $\left\|f-g_{n}\right\|$, the right panels show the norm $\|x\|$ of the solution vector.

The results are shown in Figure 9. The projection method initially converges rapidly, but levels off when an approximation error of order $1 e-8$ is reached. The collocation method, somewhat surprisingly, actually reaches machine precision for both example functions. The Matlab computed solution has an accuracy comparable to that of the explicit truncation approach for the collocation method, but it is somewhat less accurate for the projection method.

The norms of the solution vectors are shown in the right panels of Figure 9. We observe that the solution vectors may be very large, especially for the second function $f_{2}$. Yet, for increasing $n$, their norms start converging for three out of four methods. The Matlab computed solution $A \backslash B$ for the projection method does not yield small Fourier coefficients. The convergence of the norms for the other three methods is shown in more detail in Figure 10. Both explicit truncation methods yield a solution vector with a norm that approaches the optimal value $1 / \sqrt{2}\|f\|$ when $n$ increases. The Matlab computed solution $\tilde{A} \backslash \tilde{B}$ yields a solution vector with a norm that approaches $\|f\|$. We note that in this case, by default, Matlab attempts to produce the sparsest solution to $\tilde{A} x=\tilde{B}$, rather than the solution with minimal norm. 


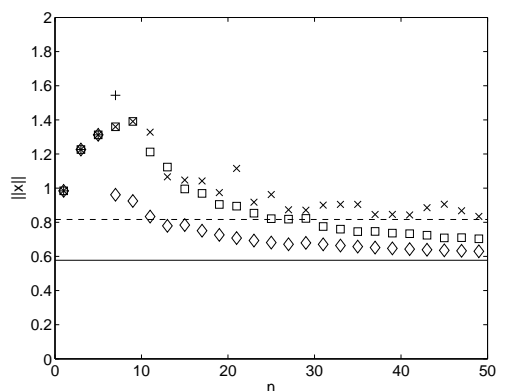

(a) $f 1$ : norm of solution vector

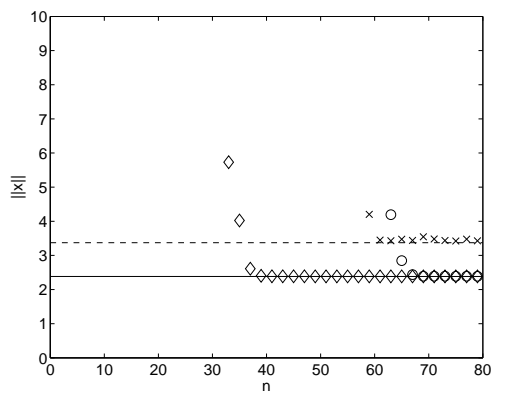

(b) $f 2$ : norm of solution vector

Figure 10: The panels (b) and (d) of Figure 9 are shown in a different scale. Also shown is the norm $\|f\|$ (dashed line) and the value $1 / \sqrt{2}\|f\|$ (solid line).

\section{Computing the exact solution}

It is numerically unstable to represent the exact solution $g_{n}$ to Problem 1.1 in the basis $D_{n}$. However, the representation in terms of orthogonal polynomials is stable. There is a general algorithm to compute polynomial expansions in a stable manner, based on the associated Gaussian quadrature. In this section we describe its application to the Fourier extension problem.

\subsection{Gaussian quadrature}

Both families of orthogonal polynomials $T_{m}^{h}$ and $U_{m}^{h}$ have associated families of Gaussian quadrature. Denote by $\left\{y_{j}^{F K}\right\}_{j=1}^{m}$ the roots of the half-range Chebyshev polynomials of the first kind $T_{m}^{h}(y)$. Note that all roots lie in the interval $[0,1]$ and that the associated weights are positive [10]. The weights satisfy

$$
\sum_{j=1}^{m} w_{j}^{F K}\left(y_{j}^{F K}\right)^{j}=\frac{4}{\pi} \int_{0}^{1} y^{j} T_{j}^{h}(y) \frac{1}{\sqrt{1-y^{2}}} \mathrm{~d} y, \quad k=0, \ldots, 2 m-1,
$$

i.e., the quadrature rule is exact for polynomials up to degree $2 m-1$. The weights can be computed efficiently in a numerically stable manner based on the recurrence coefficients of the orthogonal polynomials [11].

Similarly, denote by $\left\{y_{j}^{S K}\right\}_{j=1}^{m}$ the roots of the half-range Chebyshev polynomials of the second kind $U_{m}^{h}(y)$. The associated weights satisfy

$$
\sum_{j=1}^{m} w_{j}^{S K}\left(y_{j}^{S K}\right)^{j}=\frac{4}{\pi} \int_{0}^{1} y^{j} U_{j}^{h}(y) \sqrt{1-y^{2}} \mathrm{~d} y, \quad k=0, \ldots, 2 m-1 .
$$

It is interesting to point out that the first kind of quadrature rule also leads to a quadrature rule on $[-1,1]$ that is exact for all trigonometric functions in $G_{2 m-1}$. Define $2 m$ quadrature points and weights as follows,

$$
\begin{cases}x_{j}^{T R} & =\frac{2}{\pi} \cos ^{-1} y_{j}^{F K} \\ x_{j+m}^{T R} & =-\frac{2}{\pi} \cos ^{-1} y_{j}^{F K} \\ w_{j}^{T R} & =\frac{1}{2} w_{j}^{F K} \\ w_{j+m}^{T R} & =\frac{1}{2} w_{j}^{F K}\end{cases}
$$


The transformation $x=\frac{2}{\pi} \cos ^{-1} y$ is applied to the points $y_{j}^{F K}$. This transformation maps the interval $[0,1]$ to itself. However, the resulting rule may only be applied to even functions. The quadrature points are therefore mirrorred to $[-1,0]$, with halved weights, to select the even part of any given function $f$. As a result, the rule is exact for all odd functions on $[-1,1]$. The quadrature approximation is 0 whenever $f(x)=-f(-x)$.

\subsection{Computing the coefficients}

The coefficients $a_{k}$ and $b_{k}$ of the exact solution (19) may be computed based on expressions (23) and (24). We have

$$
\begin{aligned}
& a_{k} \approx Q_{k}^{F K}[f]=\sum_{j=1}^{m} w_{j}^{F K} f_{e}\left(\frac{2}{\pi} \cos ^{-1} y_{j}^{F K}\right) T_{k}^{h}\left(y_{j}^{F K}\right), \\
& b_{k} \approx Q_{k}^{S K}[f]=\sum_{j=1}^{m} w_{j}^{S K} \frac{f_{o}\left(\frac{2}{\pi} \cos ^{-1} y_{j}^{S K}\right)}{\sqrt{1-\left(y_{j}^{S K}\right)^{2}}} U_{k}^{h}\left(y_{j}^{S K}\right) .
\end{aligned}
$$

Equivalent expressions are

$$
\begin{aligned}
& Q_{k}^{F K}[f]=\sum_{j=1}^{m} \frac{1}{2} w_{j}^{F K}\left[f\left(\frac{2}{\pi} \cos ^{-1} y_{j}^{F K}\right)+f\left(-\frac{2}{\pi} \cos ^{-1} y_{j}^{F K}\right)\right] T_{k}^{h}\left(y_{j}^{F K}\right), \\
& Q_{k}^{S K}[f]=\sum_{j=1}^{m} \frac{1}{2} w_{j}^{S K}\left[\frac{f\left(\frac{2}{\pi} \cos ^{-1} y_{j}^{S K}\right)}{\sqrt{1-\left(y_{j}^{S K}\right)^{2}}}-\frac{f\left(-\frac{2}{\pi} \cos ^{-1} y_{j}^{F K}\right)}{\sqrt{1-\left(y_{j}^{S K}\right)^{2}}}\right] U_{k}^{h}\left(y_{j}^{S K}\right) .
\end{aligned}
$$

\subsection{Convergence}

Let us determine the number of quadrature points necessary to compute the coefficients $a_{k}$ and $b_{k}$, such that the computed solution converges at the same rate as the exact solution. To that end, write the function $f$ as

$$
f(x)=g_{n}(x)+R(x),
$$

where $g_{n}(x)$ is the exact solution to Problem 1.1 and $R(x)$ is a remainder term. Recall that $g_{n}(x)$ has the form (19).

Theorem 5.1. Let $a_{k}$ be approximated by $Q_{k}^{F K}[f]$ with $m=n+1$ and let $b_{k}$ be approximated by $Q_{k}^{S K}[f]$ with $m=n$. If $f(x)$ can be written as (50), with $R(x)$ continuously differentiable, then there exist constants $c_{1}, c_{2}>0$ such that

$$
\begin{aligned}
\left|Q_{k}^{F K}[f]-a_{k}\right| & \leq c_{1} n \sqrt{k}\|R\|_{\infty}, \\
\left|Q_{k}^{S K}[f]-b_{k}\right| & \leq c_{2} n k\left\|R^{\prime}\right\|_{\infty} .
\end{aligned}
$$

Proof. The even part of $f$ can be written as

$$
f_{e}(x)=\sum_{i=0}^{n} a_{i} T_{i}^{h}\left(\cos \frac{\pi}{2} x\right)+R_{e}(x)
$$


where $R_{e}(x)=\frac{1}{2}(R(x)+R(-x))$ is the even part of $R$. We have

$$
Q_{k}^{F K}[f]=Q_{k}^{F K}\left[f_{e}\right]=\sum_{i=0}^{n} a_{i} Q_{k}^{F K}\left[T_{i}^{h}\left(\cos \frac{\pi}{2} \cdot\right)\right]+Q_{k}^{F K}\left[R_{e}\right] .
$$

Consider first the sum in this expression. We have

$$
Q_{k}^{F K}\left[T_{i}^{h}\left(\cos \frac{\pi}{2} \cdot\right)\right]=\sum_{j=1}^{m} w_{j}^{F K} T_{k}^{h}\left(y_{j}^{F K}\right) T_{i}^{h}\left(y_{j}^{F K}\right) .
$$

The quadrature rule is applied to a polynomial of degree $k+i$. Since $k \leq n$ and $i \leq n$, the maximal degree is $2 n$. Hence, letting $m=n+1$, the quadrature rule is exact for these polynomials. We find that $Q_{k}^{F K}\left[T_{i}^{h}\left(\cos \frac{\pi}{2} \cdot\right)\right]=\delta_{k-i}$ and

$$
Q_{k}^{F K}[f]=a_{k}+Q_{k}^{F K}\left[R_{e}\right] .
$$

Next, consider the remainder term

$$
\begin{aligned}
Q_{k}^{F K}\left[R_{e}\right] & =\sum_{j=1}^{m} w_{j}^{F K} R_{e}\left(\frac{2}{\pi} \cos ^{-1} y_{j}^{F K}\right) T_{k}^{h}\left(y_{j}^{F K}\right) \\
& \leq\left\|R_{e}\right\|_{\infty} \sum_{j=1}^{m} w_{j}^{F K}\left|T_{k}^{h}\left(y_{j}^{F K}\right)\right| .
\end{aligned}
$$

It follows from Theorem 3.5 that the polynomials grow at most like $\sqrt{k}$. Since $\left\|R_{e}\right\|_{\infty} \leq\|R\|_{\infty}$, the first result follows.

The reasoning for the odd part of $f$ is analogous, except for the remainder term. We have

$$
Q_{k}^{S K}\left[R_{o}\right]=\sum_{j=1}^{m} w_{j}^{S K} \frac{R_{o}\left(\frac{2}{\pi} \cos ^{-1} y_{j}^{S K}\right)}{\sqrt{1-\left(y_{j}^{S K}\right)^{2}}} U_{k}^{h}\left(y_{j}^{S K}\right) .
$$

The denominator is unbounded if $y_{j}^{S K}$ approaches 1 . However, since $R_{o}(x)$ is odd, we may write $R_{o}(x)=x h(x)$. The function

$$
\frac{\frac{2}{\pi} \cos ^{-1} y}{\sqrt{1-y^{2}}} h\left(\frac{2}{\pi} \cos ^{-1} y\right)
$$

is bounded on $[0,1]$. Note that $h(0)=R_{o}^{\prime}(0)=R^{\prime}(0)$ and, since $R_{o}(0)=0$, we may bound $h(x)$ by $\left\|R^{\prime}(x)\right\|_{\infty}$. From Theorem 3.5 , the polynomials grow at most like $k$. The second result follows.

Theorem 5.1 states that one should choose $n+1$ quadrature points for the computation of $a_{k}, k=0, \ldots, n$, and $n$ quadrature points for the computation of $b_{k}, k=0, \ldots, n-1$. If explicit expressions for $f_{e}$ and $f_{o}$ are available, then one requires $2 n+1$ function evaluations to compute these $2 n+1$ coefficients, using expressions (46)-(47). Note that evaluations of $f_{e}$ and $f_{o}$ may be reused for varying $k$. If such expressions are not available, then one requires $4 n+2$ evaluations of $f$ using expressions (48)-(49). The total computational complexity in both cases scales as $O\left(n^{2}\right)$. There is unfortunately no simple connection 
to the FFT for constructing the expansion in $O(n \log n)$ computations as in the case of Chebyshev expansions [24].

The convergence rate of the quadrature scheme is dictated by the results of $\S 3$ on the possible decay rate of the remainder term $R$ for increasing $n$. Compared to these results, the loss by a factor of $n^{3 / 2}$ or $n^{2}$ is observed, which is due to the growth of the orthogonal polynomials in $[-1,1]$. These bounds are rather pessimistic, since the polynomials are, in fact, bounded in the interior of $[0,1]$. Yet, they already show that an exponential rate of convergence can be maintained up to algebraic factors.

\subsection{Numerical results}

All quadrature rules were computed numerically as follows. The three term recurrence coefficients of the two families of orthogonal polynomials were computed to high precision in Maple. The corresponding Gaussian quadrature rules were then computed based on the algorithm in [11], which efficiently computes the eigenvalues of a tridiagonal matrix. The latter computation was performed in double precision in Matlab.

Several computational results were already shown earlier in this paper. The exact solution for the functions $f(x)=2 x^{2}+3 x+1$ and $f(x)=\cos \cos \frac{\pi}{2} x+$ $\sin \sin \frac{\pi}{2} x$ was shown in Figure 4 . Note that the quadrature approximation agrees with the theoretical convergence rate $E^{-n}$ for the former function, and that the quadrature approximation converges faster than exponential for the latter. Accuracy close to machine precision was shown for $f(x)=x$ in Figure 6 and for $f(x)=\frac{1}{1+2 x^{2}}$ in Figure 7 .

We supplement these examples with a small comparison of several quadrature rules. In particular, we compare classical Gaussian quadrature, ClenshawCurtis quadrature and the newly constructed Fourier extension quadrature. For the latter, we use the quadrature formula given by (45).

Gaussian quadrature and Clenshaw-Curtis quadrature were compared extensively in [23]. We added Fourier extension quadrature to six examples given in that paper. The results are shown in Figure 11. The first four examples are analytic functions, the fifth is $C^{\infty}$ and the final one is $C^{2}$. One observes in panel (a) that Fourier extension quadrature is not exact for polynomials. Panel (b) shows slightly slower convergence of Fourier extension quadrature for an entire function, because its convergence rate is bounded. Fourier extension apparently outperforms the other methods for example (d) with nearby poles. Still, the differences are small and all results are quite comparable.

Results are quite different however when integrating oscillatory functions. Figure 12 shows the quadrature approximation for $\cos (40 x)$, the Bessel function of the first kind $J_{0}(30 x)$ and the function $\cos \left(25 x^{2}\right)$. In the first two cases, Fourier extension quadrature converges faster than Gaussian quadrature and much faster than Clenshaw-Curtis quadrature. Note however that the function $J_{0}(30 x)$ has harmonic oscillations like a cosine. The function $\cos 25 x^{2}$ has oscillations that are less harmonic. In that case, Fourier extension quadrature and Gaussian quadrature perform very similarly, but Clenshaw-Curtis is still significantly less accurate. For completeness, we like to point out that significantly more efficient methods exist to evaluate oscillatory integrals (see, e.g., [15]).

Interestingly, the convergence plots of Clenshaw-Curtis quadrature in Figure 12 exhibit a kink for each of the three examples. This phenomenon was 
described and analyzed in [25] for functions with a singularity in the complex plane. It was concluded that Clenshaw-Curtis quadrature converges at the same rate as Gaussian quadrature up to a certain $n$, depending on the location of the nearest singularity. However, the three examples given here are entire functions.

\section{Concluding remarks}

It is perhaps not surprising that non-periodic functions can be represented as a trigonometric series by altering the periodicity. It is surprising however, at least in our opinion, that such series can be exponentially accurate. We focused in this paper on a constructive convergence theory for such series, which led to Chebyshev-like orthogonal polynomials.

Two associated numerical methods were studied. A numerical least squares method leads to hideously ill-conditioned matrices and produces a solution that does not resemble the exact least squares solution to Problem 1.1. Yet, it approximates $f$ to machine precision on the interval $[-1,1]$. The true solution can also be computed by exploiting the link with orthogonal polynomials and the associated Gaussian quadrature.

It appears that the results in this paper can be generalized to several bounded domains in higher dimensions. Preliminary results suggest that exponentially accurate Fourier series can be constructed for functions defined on triangles, tetrahedra and even higher-dimensional simplices. The path towards generalization lies in the connection to eigenfunctions of the Laplacian and in the use of symmetries of these domains. This is an active topic of further research.

\section{Acknowledgements}

The author would like to thank Arieh Iserles and Syvert Nørsett for introducing him to Laplace-Neumann expansions, which eventually led to the research in this article. The author also acknowledges useful comments and suggestions by John Boyd, Oscar Bruno and Rodrigo Platte.

\section{References}

[1] S. N. Bernstein. Collected Works vol. 2. Akademii nauk SSSR, Moscow, 1954.

[2] J. P. Boyd. Chebyshev and Fourier spectral methods. Courier Dover Publications, 2001.

[3] J. P. Boyd. A comparison of numerical algorithms for Fourier extension of the first, second, and third kinds. Journal of Computational Physics, 178(1):118-160, 2002.

[4] J. P. Boyd. Fourier embedded domain methods: extending a function defined on an irregular region to a rectangle so that the extension is spatially periodic and $c^{\infty}$. Appl. Math. Comput., 161:591-597, 2005. 
[5] O. P. Bruno, Y. Hana, and M. M. Pohlman. Accurate, high-order representation of complex three-dimensional surfaces via Fourier continuation analysis. Journal of Computational Physics, 227(2):1094-1125, 2007.

[6] I. Daubechies. Ten lectures on wavelets. SIAM, Philadelphia, 1992.

[7] D. Donoho. For most large underdetermined systems of linear equations the minimal $l_{1}$-norm solution is also the sparsest solution. Comm. Pure Appl. Math., 59(6):797-829, 2006.

[8] R. J. Duffin and A. C. Schaeffer. A class of nonharmonic Fourier series. Transactions of the American Mathematical Society, 72(2):341-366, 1952.

[9] C. Fefferman. Interpolation and extrapolation of smooth functions by linear operators. Rev. Mat. Iberoamericana, 21(1):313-348, 2005.

[10] W. Gautschi. Orthogonal polynomials: computation and approximation. Clarendon Press, Oxford, 2004.

[11] G. H. Golub and J. H. Welsch. Calculation of Gauss quadrature rules. Math. Comp., 23(106):221-230, 1969.

[12] D. Gottlieb and C.-W. Shu. On the Gibbs phenomenon and its resolution. SIAM Rev., 39(4):644-668, 1997.

[13] M. R. Hestenes. Extension of the range of a differentiable function. Duke Math. J., 8(1):183-192, 1941.

[14] D. Huybrechs, A. Iserles, and S. P. Nørsett. From high oscillation to rapid approximation IV: Accelerating convergence. Technical Report NA2007/07, University of Cambridge, 2007.

[15] D. Huybrechs and S. Vandewalle. On the evaluation of highly oscillatory integrals by analytic continuation. SIAM J. Numer. Anal., 44(3):10261048, 2006.

[16] A. Iserles and S. P. Nørsett. From high oscillation to rapid approximation I: Modified Fourier expansions. Technical Report 2006/NA05, University of Cambridge, 2006.

[17] A. Iserles and S. P. Nørsett. From high oscillation to rapid approximation II: Expansions in polyharmonic eigenfunctions. Technical Report 2006/NA07, University of Cambridge, 2006.

[18] A. Iserles and S. P. Nørsett. From high oscillation to rapid approximation III:Multivariate expansions. Technical Report 2007/NA01, University of Cambridge, 2007.

[19] C. L. Lawson and R. J. Hanson. Solving least squares problems. Classics in Applied Mathematics. SIAM, Philadelphia, 1996.

[20] J. C. Mason and D. C. Handscomb. Chebyshev polynomials. Chapman and Hall/CRC, 2003.

[21] E. M. Stein and R. Shakarchi. Fourier analysis: an introduction. Princeton University Press, 2003. 
[22] E. Tadmor. Filters, mollifiers and the computation of the Gibbs phenomenon. Acta Numer., 16:305-378, 2007.

[23] L. N. Trefethen. Is Gauss quadrature better than Clenshaw-Curtis? SIAM Rev., 50(1):67-87, 2008.

[24] J. Waldvogel. Fast construction of the Fejér and Clenshaw-Curtis quadrature rules. BIT, 46:195-202, 2006.

[25] J. A. C. Weideman and L. N. Trefethen. The kink phenomenon in Fejér and Clenshaw-Curtis quadrature. Numer. Math., 107(4):707-727, 2007.

[26] H. Whitney. Analytic extensions of differentiable functions defined in closed sets. Trans. Amer. Math. Soc., 36(1):63-89, 1934. 


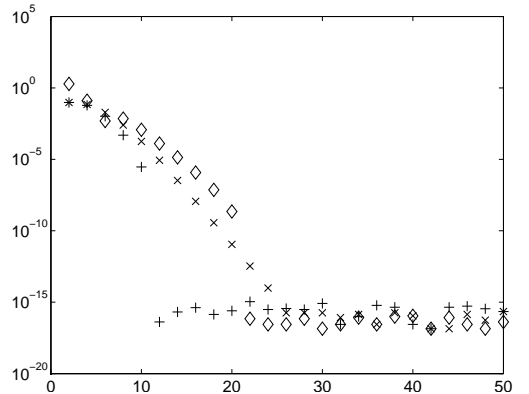

(a) $f(x)=x^{20}$

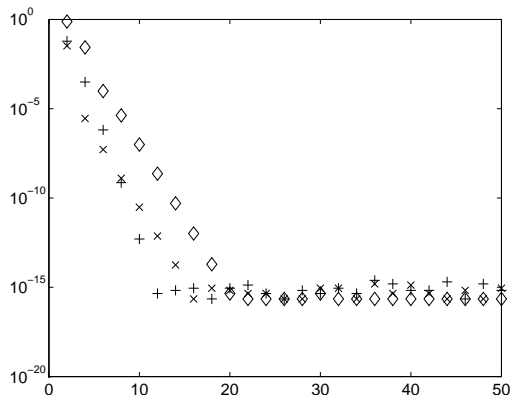

(c) $f(x)=\exp \left(-x^{2}\right)$

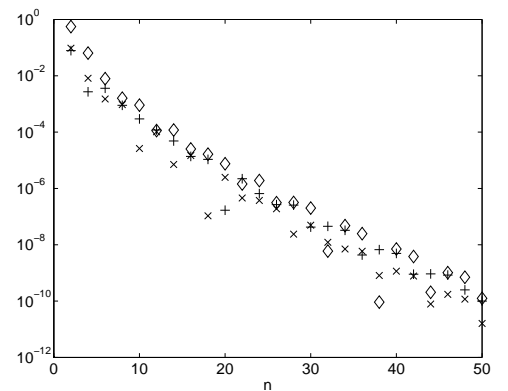

(e) $f(x)=\exp \left(-1 / x^{2}\right)$

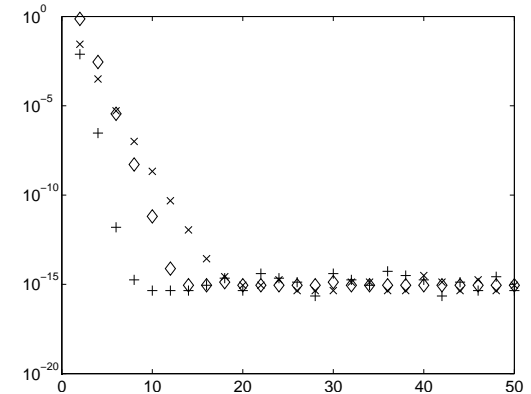

(b) $f(x)=\exp (x)$

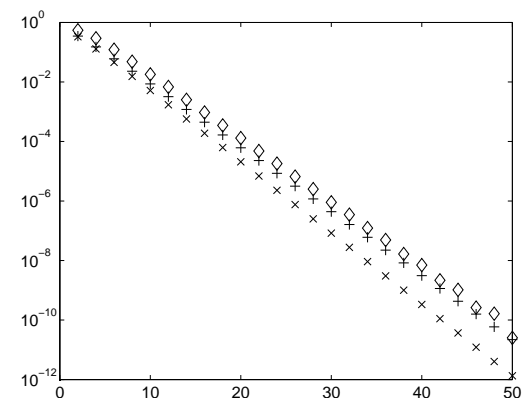

(d) $f(x)=\frac{1}{1+16 x^{2}}$

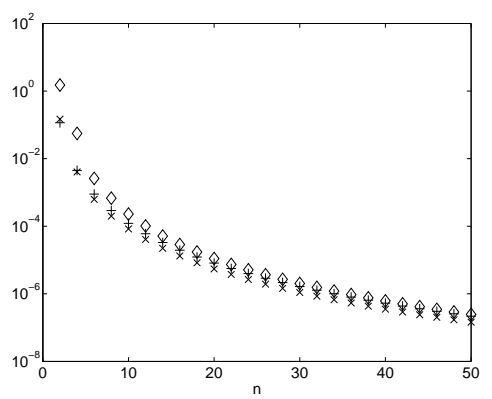

(f) $f(x)=|x|^{3}$

Figure 11: Comparison of Gaussian quadrature $(+)$, Clenshaw-Curtis quadrature $(\diamond)$ and Fourier extension quadrature $(\times)$ on $[-1,1]$. 


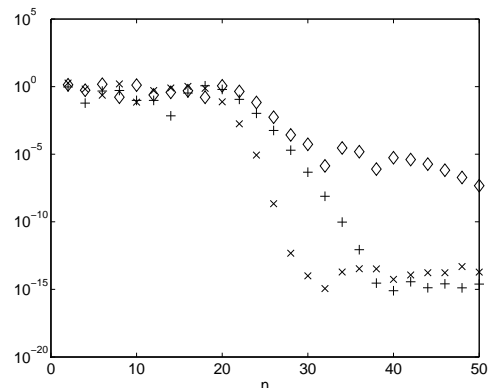

(a) $f(x)=\cos 40 x$

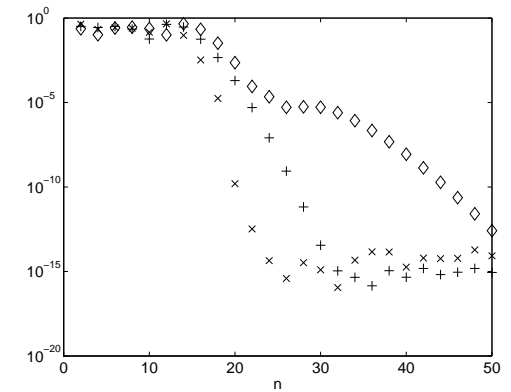

(b) $f(x)=J_{0}(30 x)$

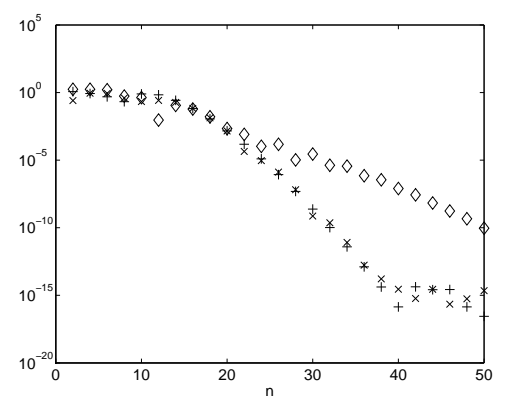

(c) $f(x)=\cos 25 x^{2}$

Figure 12: Comparison of Gaussian quadrature $(+)$, Clenshaw-Curtis quadrature $(\diamond)$ and Fourier extension quadrature $(\times)$ on $[-1,1]$. 